\title{
Genetic learning as an explanation of stylized facts of foreign exchange markets
}

\author{
Thomas Lux $^{\mathrm{a}, *}$, Sascha Schornstein ${ }^{\mathrm{b}}$ \\ a Department of Economics, University of Kiel, Olshausenstr. 40, 24118 Kiel, Germany \\ ${ }^{\mathrm{b}}$ Deutsche Bank, Winchester House, London EC2N 2DB, UK
}

Received 22 October 2002; received in revised form 10 July 2003; accepted 16 February 2004

Available online 4 July 2004

\begin{abstract}
This paper revisits the Kareken-Wallace model of exchange rate formation in a two-country overlapping generations world. Following the seminal paper by Arifovic [Journal of Political Economy 104 (1996) 510] we investigate a dynamic version of the model in which agents' decision rules are updated using genetic algorithms. Our main interest is in whether the equilibrium dynamics resulting from this learning process helps to explain the main stylized facts of free-floating exchange rates (unit roots in levels together with fat tails in returns and volatility clustering). Our time series analysis of simulated data indicates that for particular parameterizations, the characteristics of the exchange rate dynamics are, in fact, very similar to those of empirical data. The similarity appears to be quite insensitive with respect to some of the ingredients of the genetic algorithm (i.e. utility-based versus rank-based or tournament selection, binary or real coding). However, appearance or not of realistic time series characteristics depends crucially on the mutation probability (which should be low) and the number of agents (not more than about 1000). With a larger population, this collective learning dynamics looses its realistic appearance and instead exhibits regular periodic oscillations of the agents' choice variables.

(C) 2004 Elsevier B.V. All rights reserved.
\end{abstract}

JEL classification: D83; D 84; F31

Keywords: Learning; Genetic algorithms; Exchange rate dynamics

\footnotetext{
* Corresponding author.

E-mail address: lux@bwl.uni-kiel.de (T. Lux).
} 


\section{Introduction}

Foreign exchange markets as well as other financial markets are characterized by a number of striking ubiquitous time series features. Most prominently, $(\log )$ exchange rates seem to be non-stationary while their first differences are stationary. More precisely, unit root tests are typically unable to reject the null hypothesis of a first-order autoregressive process with a coefficient equal to unity. This finding squares with the well-known result of Meese and Rogoff (1983) that random walk forecasts produce a lower mean-squared error in out-of-sample prediction than reduced-form structural models of macroeconomic fundamentals. It has been argued that these findings can be explained by speculative efficiency of foreign exchange markets, which simply means one interprets the foreign exchange market as an informationally efficient market in the sense of the Efficient Market Hypothesis (cf. Bilson, 1981).

While from this perspective the unit root property may not be viewed as a conundrum, other well-known features have defied straightforward explanations until recently. The most pervasive ones are the fat-tail property of relative price changes and the clustering of volatility in these time series. Traces of these features are easily recognizable in all records of high-frequency data (probably up to weekly frequency) of foreign exchange markets (to our knowledge, without any known exception). The fat-tail property implies that the unconditional distribution of daily returns (as well as those of higher and somewhat lower frequency) has more probability mass in the tails and the center than the standard Normal distribution. This also means that extreme changes occur more often than would be expected under the assumption of Normality of relative daily price changes. Volatility clustering means that periods of quiescence and turbulence tend to cluster together. Hence, the volatility (conditional variance) of exchange rate changes is not homogeneous over time, but is itself subject to temporal variation.

Explanations of these stylized facts have been elusive until very recently. Perhaps, the silence of economic theory on this issue is not too surprising given that the above regularities are features of time series as a whole and, hence, could only be explained by dynamic models of the evolution of the trading process in the pertinent market. From the viewpoint of informational efficiency, the characteristics of returns would, of course, have to be explained by similar characteristics of the news arrival process, but due to the unobservability of the later, this hypothesis can hardly be subjected to econometric scrutiny. As an alternative, some authors have recently argued that fat tails and clustered volatility can be obtained as a result of interactions of heterogeneous economic agents. Examples of this emergent literature include Lux and Marchesi (1999, 2000), Chen et al. (2001), Kirman and Teyssière (2002), Gaunersdorfer and Hommes (2000), Chiarella and He (2001), Iori (2002) and Bornholdt (2001). Lux and Marchesi, Gaunersdorfer and Hommes, and Chiarella and He have models of fundamentalist — chartist interaction in financial markets which give rise to realistic behavior of the resulting time series (in terms of the above stylized facts). In Lux and Marchesi and Gaunersdorfer and Hommes, the authors try to provide some hints of general mechanisms that could generate these time series properties irrespective of the details of their exemplary models. In the former case, it is a critical behavior of the dynamics in the vicinity of a continuum of equilibria with an indeterminate composition of the population in terms of strategies pursued by individuals. Gaunersdorfer and Hommes get 


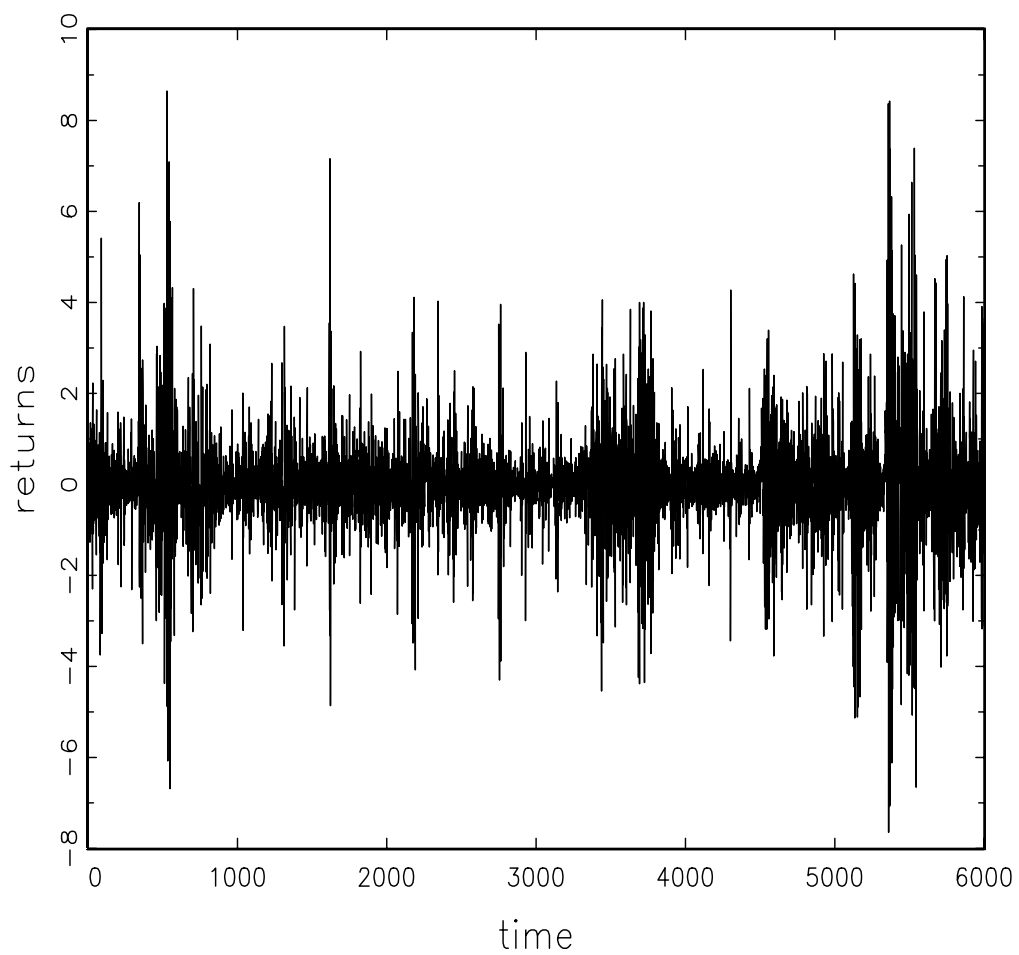

Fig. 1. A typical 'realistic' series of returns from a simulated economy with a binary-coded GA population of 100 agents. For economic parameters, see main text. GA parameters are: $p_{\text {mut }}=0.01$ and $p_{\text {cross }}=0.6$.

similar dynamics from a model with co-existing attractors in which noise leads to switches between different states. Still different mechanisms prevail in Iori (2002) and Bornholdt (2001) who use lattice-based structures for modeling the interactions among traders. Interestingly, a recent paper by Arifovic and Gencay (2000) on an artificial currency market with genetic learning of strategies also suggests emergence of realistic features of the resulting exchange rate dynamics (cf. Fig. 1). However, they do not provide a detailed analysis concerning the above properties. One of the aims of this paper is to fill this gap. In particular, we will try to quantitatively assess the degree of fat tailedness and volatility clustering this model generates. We are also interested in the sensitivity of these quantitative measures with respect to key parameters of the model. To get an impression of the sensitivity with respect to parameter variations, we will try to figure out how the time series properties depend on the genetic algorithm parameters and the number of agents populating the market (as will probably become clear in the presentation of the model, the values of the few economic variables of the model are less important in this respect). We then relate our findings to those obtained for other models of artificial financial markets and try to provide an explanation for the crucial importance of the number of individuals for the qualitative outcome of the model. 
When investigating the sensitivity of the results, we are particularly interested in (i) the sensitivity with respect to the details of the learning dynamics, and (ii) the influence of the number of agents. Sensitivity with respect to the GA design is investigated by using different implementations of the typical reproduction, cross-over and selection operators, varying the parameters used for these operators, and applying real-coded GAs besides the traditional binary coded ones. As far as we can see, this is the first application of real-coded GAs in economics. Since there are no rigorous guidelines for the choice of one particular form of encoding, such a sensitivity analysis gives some insight into the generality of results obtained from GA learning and, in fact, it turned out that results were quite robust with respect to the coding scheme. Our interest in the effects of the size of the market derives from some puzzling earlier findings. Namely, a number of studies have revealed that existing multi-agent models of financial markets loose their realistic time series properties when increasing the number of agents (Egenter et al., 1999; Yeh, 2001; Challet and Marsili, 2002). Since published work on artificial markets with GA learning has used only a very limited number of agents, typically below 100, it seems worthwhile to explore the behavior of larger economies.

Apart from our interest in explaining stylized facts, the present paper also fits into the broader framework of evolutionary models of financial markets. Like most of the above references, we consider adaptive choice of strategies by our agents governed by some kind of fitness criterion. Here fitness is determined by the utility obtained by agents pursuing a certain strategy. Alternative fitness criteria in the literature include the performance of some predictors on which a trading strategy depends or its (short-term) profitability. Typical examples of this literature are the 'adaptive belief systems' of Brock and Hommes (1997) and Gaunersdorfer and Hommes (2000). However, our set-up differs from theirs in various aspects: (i) instead of an isolated financial market we consider a two-country general equilibrium framework, (ii) instead of modeling the evolutionary strategy choice via a discrete choice framework we apply genetic algorithms as an evolutionary algorithm for behavioral adaptations, and (iii) we allow for as many strategies as can be formulated with a given GA design (i.e. a large number or continuum, see below) instead of focusing on typical examples like chartist-fundamentalist interaction. These differences notwithstanding, our aim of investigating double limits of large populations operating within a large strategy space, is, in fact, very similar to the analysis of large type limits in Brock et al. (2003).

Another related strand of evolutionary finance literature looks at the development of wealth attained by different competing strategies instead of allowing agents to switch between strategies based on a comparison of their performance in the short or medium run. Originating from Blume and Easley's (1992) paper, the ensuing literature on the 'market selection hypothesis' has come out with some stark results: in particular, it has been shown that in a conventional financial market with many agents, the highest growth in wealth is realized by agents whose saving rate is as least as high as the market saving rate and whose investment shares in different assets are equal to the expected relative pay-offs of these assets. Under uncertain subjective beliefs about pay-offs, Bayesian learners dominate other types of adaptive behavior (Blume and Easley, 1992). While Blume and Easley had shown the dominance of the 'betting your belief' strategy in the case of Arrow securities, Hens and Schenk-Hoppé (2003) have been able to generalize this result for arbitrary pay-off structures. They also show that CAPM is able to mimic the optimal portfolio rule. The 
result on the dominance of Bayesian learners is sharpened by Sandroni (2003), who shows that Bayesian learning with a uniform prior dominates maximum likelihood models when available information is limited. In contrast to the first strand of literature, the 'market selection hypothesis' deals with the success of strategies, not agents. However, survey studies show that investors often switch between different strategies and that their choice of trading strategy is governed by short-run success criteria (short termism). It would, therefore, be worthwhile to consider the endogenous development of agents' wealth in models allowing for switching between strategies. A first attempt at combining wealth dynamics and switching between strategies is provided by Chiarella and He (2002).

The rest of the paper proceeds in the following steps: Section 2 will introduce the underlying model of the foreign exchange market, the well-known Kareken-Wallace two-country overlapping generations model. Section 3 gives details on the genetic algorithms which we apply to model the learning of our agents. In Section 4, we review the statistics used for assessing how realistic the model's output is. Section 5 presents the results of extensive Monte Carlo work, and Section 6 tries to provide an explanation for the surprising behavior that we find in the case of a very large population. Section 7 concludes.

\section{The Kareken and Wallace OLG economy}

As a version of the Kareken and Wallace (1982) two country model, the underlying economic structure is extremely simple: at each date $t$, one-half of the entire population is replaced by a new generation (the young), while the remaining members are in the second and final period of their lives (the old) and will be replaced in the next period by another young generation. Each agent is endowed with $w_{1}$ units of a homogeneous good in its first period and with $w_{2}$ units in the second period of its live. There is neither production nor inheritance of goods. Intertemporal consumption smoothing can be achieved via money holdings of currency of the home and foreign country.

With identical preferences of all agents, $U\left(c_{i}(t), c_{i}(t+1)\right)$, their consumption plans and money demand are derived from

$$
\max U\left(c_{i}(t), c_{i}(t+1)\right)
$$

subject to:

$$
c_{i}(t) \leq w_{1}-\frac{m_{i, 1}(t)}{p_{1}(t)}-\frac{m_{i, 2}(t)}{p_{2}(t)}
$$

and

$$
c_{i}(t+1) \leq w_{2}+\frac{m_{i, 1}(t)}{p_{1}(t+1)}+\frac{m_{i, 2}(t)}{p_{2}(t+1)},
$$

with $c_{i}(t), m_{i, 1}(t)$ and $m_{i, 2}(t)$ : consumption and money holdings of agent $i(i=1, \ldots, N)$ at time $t, w_{1}$ and $w_{2}$ the homogeneous endowment levels, and $p_{1}(t)$ and $p_{2}(t)$ the price levels in both countries at time $t$. Note that with this set-up, it even does not matter, how many of the agents are citizens of countries 1 or 2 as their economic decisions are not affected by their geographic location. 
Assuming that nominal money supply $H_{1}$ and $H_{2}$, is constant, and denoting by $s_{i}(t)$ overall 'savings' of individual $i$, the price levels at time $t$ are determined by:

$$
p_{1}(t)=\frac{H_{1}}{\sum_{i} f_{i}(t) s_{i}(t)}, \quad p_{2}(t)=\frac{H_{2}}{\sum_{i}\left(1-f_{i}(t)\right) s_{i}(t)}
$$

with

$$
f_{i}(t)=\frac{m_{i, 1}(t) / p_{1}(t)}{s_{i}(t)}
$$

the fraction of currency 1 in agent $i$ 's portfolio at time $t$.

The exchange rate, $e(t)$, between both countries is, then, obtained as $e(t)=p_{1}(t) / p_{2}(t)$.

It is straightforward to spell out some results on possible equilibria (steady states) of this model. Using $c_{i}(t)$ and $f_{i}(t)$ as choice variables of our agents, it is immediately obvious that a stationary solution requires the rates of returns on both currencies to be equal, i.e. $p_{1}(t) / p_{1}(t+1)=p_{2}(t) / p_{2}(t+1)$.

Simple inspection shows that such a steady state has the following properties:

(1) the exchange rate is constant over time: $e(t+1)=e(t)=e^{*}$. However, the level of the exchange rate in equilibrium, $e^{*}$, is indeterminate and may be any value on the half-line, $e^{*} \in(0, \infty)$.

(2) Given the equality of returns form both currencies, the equilibrium composition of each agent's portfolio is indeterminate as well and $f_{i}{ }^{*}$, therefore, might assume any admissible value $f_{i}^{*} \in[0,1]$. Note that we do not have to assume identical portfolio choices of the agents in equilibrium. Any constant distribution of the $f_{i}^{*}$ 's over generations would be consistent with constant rates of returns and a constant exchange rate. We could even allow for certain changes of the distribution of the $f_{i}{ }^{*}$ 's: equilibrium would still prevail as long as the mean value of their distribution remains the same over time.

(3) Since the rates of returns from both assets are identical in equilibrium, optimal consumption plans do not depend on the portfolio composition. With a well-behaved utility function, utility maximizing choice $c_{i}{ }^{*}$ will typically be unique and identical for all individuals with the same utility function and endowment structure. For example, with utility functions $U_{i}=c_{i}(t) c_{i}(t+1)$ and constant money supply in both countries, rates of return would be equal to one in equilibrium and $c_{i}{ }^{*}$ would be $0.5\left(w_{1}+w_{2}\right)$ for all individuals.

The non-uniqueness of the equilibrium exchange rate in this type of model derives from the absence of typical macroeconomic fundamentals of monetary models. In view of the evidence on unit roots in empirical data, this feature has been emphasized as an advantage of their model by Kareken and Wallace. One could indeed imagine that added random fluctuations could easily produce a unit root dynamics, since random disturbances could lead to a random motion of the exchange rate along the continuum of possible equilibria (every time, the equilibrium is distorted by random shocks, the exchange rate would settle at a new equilibrium). However, non-uniqueness of equilibria also raises the questions of selection of equilibria and coordination of agents. These questions have been taken up first by Sargent (1993) who modeled learning via stochastic approximation algorithms. Later on Arifovic (1996) considered GA learning in the Kareken-Wallace framework. Looking 
at the evolution of returns instead of the level of the exchange rate Arifovic and Gencay (2000) recovered realistic features in the continuing fluctuations of the resulting dynamics.

\section{Genetic algorithm learning}

Genetic algorithms have been introduced by Holland (1975) as a stochastic search algorithm for numerical optimization. This approach uses operations similar to genetic processes of biological organisms to develop better solutions of an optimization problem from an existing 'population' of randomly initiated candidate solutions. Typically, the proposed solutions have been encoded in strings (chromosomes) using a binary alphabet (see Dawid, 1999 for a general introduction). This is also the structure of the GAs applied in Arifovic and Gencay (2000). Each individual's decisions are encoded in a binary string of length $l=30$, whose first 20 elements encode first-period consumption and whose remaining 10 entries encode the fraction of currency one in his portfolio. ${ }^{1}$ With $a_{i, t}^{k}$ denoting the value at the $k$-th position of the string ( 0 or 1$)$, the binary string is translated into a real-valued number in the following way:

$$
c_{i}(t)=\sum_{k=1}^{20} a_{i, t}^{k} \frac{2^{k-1}}{K_{1}}, \quad f_{i}(t)=\sum_{k=21}^{30} a_{i, t}^{k} \frac{2^{k-21}}{K_{2}},
$$

where $K_{1}$ and $K_{2}$ are normalizing constants to restrict the possible real values to a predetermined admissible interval.

In order to have $f_{i}(t) \in[0,1], K_{2}$ is set equal to $K_{2}=2^{10}-1$, while $c_{i}(t)$ should be within the interval $\left[0, w_{1}\right]$ to guarantee viable consumption plans. With $\mathrm{w}_{1}=10$ in Arifovic and Gencay's simulations, this amounts to $K_{1}=\left(2^{20}-1\right) / 10$.

The overlapping generations structure of the model implies an overlapping genetic algorithms structure of the evolutionary process. After each period, half of the population members have completed their life cycle. With the resulting consumption in their old age their achieved fitness (utility) can be determined and used for the genetic creation of a new pool of agents entering the economy as the young generation of the following period. ${ }^{2}$

The genetic operations applied at this stage are the following:

(1) Reproduction: from the pool of old individuals, copies are selected (with replacement) with probabilities depending on their relative fitness, i.e. on $U_{i} / \Sigma U_{i}$. Other algo-

\footnotetext{
${ }^{1}$ Choosing $l=30$ with substrings of 20 and 10 bits, respectively, we closely followed Arifovic (1996) and Arifovic and Gencay (2000). However, like with most other details of the genetic algorithm implementation, variation of these numbers did not change the qualitative characteristics of the dynamics.

2 Taking the overlapping generations, overlapping GA structure literally, one time unit in the model would correspond to about 25 years. Since we know that the stylized facts of high-frequency financial data do vanish under time aggregation and are hardly visible any longer at even the level of monthly data, this long time interval would be at odds with our aim of finding possible sources of fat tails and clustered volatility. However, economic strategies are not forwarded genetically between generations of human beings anyway, so that we prefer to simply interpret the GA as a learning device which should mimic foreign exchange traders' selection of and experimentation with different strategies. It seems worthwhile to note that results of GA dynamics and experimental results from analogous economic settings have been found to be surprisingly similar (Arifovic, 1996). This suggests that GAs do capture some salient features of learning in humans.
} 
rithms for reproduction could be chosen as well: proposals in the GA literature include rank-based reproduction in which only the rank (not the absolute fitness) determines the probability of reproduction, and tournament selection, in which one repeatedly draws $n_{1}$ (say 5) individuals from the pool and accepts the $n_{2}<n_{1}$ (say 2) with highest utility among them for the new generation. Below we report only results for fitness-based reproduction. Experiments with rank-based and tournament selection have also been carried out yielding almost identical results.

(2) Cross-over: when the pool of potential new members of a generation is complete, genetic material is exchanged between them. The simplest way is randomly selecting a pair of parent strings and swapping genetic material (bits) between both chromosomes. Here, we again follow the algorithm used by Arifovic and Gencay in selecting randomly an integer in the range of $[1,29]$ and constructing offspring by combining the genetic material from the left of this position from parent one with that from the right-hand part of parent two and vice versa. Note that the cross-over operation is carried out with a certain probability $p_{\text {cross }}$ only, while with probability $1-p_{\text {cross }}$ the offspring are unchanged copies of their parents. Alternative implementations of the cross-over operator include two-point cross-over (exchange of material in an interval between two randomly chosen bits) and uniform cross-over (the two offsprings are random recombinations of their parents' bits). Again, the whole chain of our findings reported below seems to be robust with respect to the choice of the particular cross-over operator.

(3) Mutation simply means that each position within a string is altered with a certain probability $p_{\text {mut }}$ to the other value of the binary alphabet.

(4) Finally, the election operator tests newly generated offspring before letting them enter the population. In order to avoid a decrease of the fitness of the overall population due to the genetic alteration of strategies, only those among the offspring are accepted which are at least as fit as one of their parents. If after cross-over and mutation offspring have lower fitness, exact copies of their parents are placed into the new generation.

Beside these traditional binary coded GAs, we also experimented with real-coded GAs in the present framework. A real-coded GA simply uses a real representation of the choice variables. In our case, a real-coded chromosome would, therefore, consist of a pair $\left\{c_{i}(t)\right.$, $\left.f_{i}(t)\right\}$.

Mutatis mutandis, similar genetic operations can be defined for this variant (cf. Herrera et al., 1998, for an overview on real-coded GAs). First, reproduction occurs in the same way as with binary GAs. As for cross-over, a number of alternative mechanisms have been proposed in the literature. Here, we follow Eshelman and Schaffer (1993) in picking new choice variables which are uniformly and independently drawn from an interval covering the pertinent values of the parents' chromosome. To illustrate let $\bar{c}=\max \left\{c_{A}(t), c_{B}(t)\right\}$, $\underline{c}=\min \left\{c_{A}(t), c_{B}(t)\right\}$, and $d_{c}=\bar{c}-\underline{c}$ with $c_{A}(t)$ and $c_{B}(t)$ consumption choices of parents $i$ $=A, B$. Then, the first-period consumption of offsprings is determined by uniform random draws from $\left[\underline{c}-\gamma d_{\mathrm{c}}, \bar{c}+\gamma d_{\mathrm{c}}\right]$. A similar operation yields the new portfolio fractions of the offsprings. Note that $\gamma$ is a predetermined value that allows for some 'experimentation' within regions not covered by the genetic material of the parents. Its role is also to compensate for the drift towards the mean of the admissible strategy space from a cross-over operator with $\gamma=0$. Herrera et al. (1998) show that this algorithm has better performance 
on some test problems than many alternatives. Mutation with real variables is done by using Normal random variables with mean zero and small variance to slightly change the prevailing choice variables. Election, finally, occurs in the same way as with binary coding. ${ }^{3}$

\section{Empirical benchmarks}

To see whether our foreign exchange market populated by genetically learning overlapping generations has realistic time series properties we use a battery of statistical tests.

(i) Unit root tests: a realistic market should yield an exchange rate dynamics which appears to be close to a random walk. We, therefore, perform typical tests for the presence of a unit root in our synthetic time series using the standard Dickey Fuller (DF) and Augmented Dickey Fuller (ADF) tests. The underlying data are logs of the exchange rate since from the symmetric construction of the Kareken-Wallace model we would also expect symmetry of relative changes. For the ADF test, we also included the first three differences as independent variables.

(ii) Fat-tail property: the recent econometric literature has provided a very sharp characterization of this feature. In particular, it could be shown that the decline of the probability mass in the tails follows a power-law with a coefficient that is astonishingly uniform across markets. This amounts to large returns $\left(r_{t}\right)$ following a relationship: Prob. $\left(\left|r_{t}\right|>x\right) \sim x^{-\alpha}$ with the so-called 'tail index' $\alpha$ hovering within the interval between 2 and 5. Estimation of $\alpha$ using conditional maximum likelihood is straightforward, and a wealth of supporting evidence can be found in De Vries (1994) and Dacorogna et al. (2001). As a typical example, estimation of the tail index for the DM/US\$ exchange rate with daily data ranging from 1974 to 1998 yields an estimate $\alpha=3.69$ (95\% confidence interval: 3.38-4.10) when using the 5\% largest absolute returns. The review by De Vries and the monograph by Dacorogna et al. give similar statistics for other currencies.

(iii) Volatility clustering: this feature can be characterized by autoregressive dependence in various measures of volatility. Here we also have a very precise and uniform picture from almost all available data sets. In particular, it has been found that the dependence in volatility measures like squared or absolute returns extends over very long time horizons and exhibits a hyperbolic decay of the autocovariance function: $E\left[x_{t} x_{t-\Delta t}\right] \sim \Delta t^{-\kappa}$ with $x_{t}$ : squared or absolute returns. This slow decay is in contrast to fast (exponential) decline and is also denoted as long-term dependence. Like with the fat-tail property, quantitative measurements of the decay parameter $\kappa$ give very

\footnotetext{
${ }^{3}$ On a first view, the real coded GAs seem to be a much more natural way to deal with any real-valued problem than binary GAs. However, good reasons are given in the literature to actually prefer binary coding in many applications. In particular, although at first view GAs seem to process only the particular chromosomes currently represented within the population, they actually allow for a parallel processing of many different parts of alternative solutions. This might be sensed by considering the following example: the binary coded values for 0 and 4 , i.e. 000 and 100, have two-thirds of their genetic material in common, so that it is 'easy' for the genetic operations to switch from one to the other. With real-coded GAs, 0 and 4 have nothing in common and a large succession of crossovers and mutations is needed to move from one to the other.
} 
uniform results across markets. As a benchmark for our later analysis of simulated data, we give estimates from the frequently used periodogram regression technique due to Geweke and Porter-Hudak (1983). These authors propose a method for estimating the parameter of fractional differentiation, denoted $d$ in the following, which is related to $\kappa$ via: $\kappa=1-2 d$. An estimate of $d$ significantly larger than 0 would be interpreted as evidence in favor of long memory. Inability of rejection of $d=0$ would indicate absence of long-term dependence. For the sake of illustration, daily $\mathrm{DM} / \$$ data yield (95\% confidence intervals in brackets): raw returns: $d=0.07(-0.09,0.23)$; squared returns: $d=0.24(0.08,0.40)$, absolute returns: $0.29(0.13,0.45)$. Again, a glance at, for example, Dacorogna et al. shows that these figures are quite representative for foreign exchange data (as well as for financial data in general). The inability of rejection of $d=0$ for raw returns, of course, squares well with the unit root property of log exchange rates. The finding of a higher level of persistence in absolute returns rather than squared returns is also quite universal and has by itself motivated a large body of recent econometrics literature.

\section{Pseudo-empirical results}

Tables 1-6 present the results of a large number of experiments with various versions of our artificial economy. Tables 1-3 show results for binary coded GAs, while Tables 4-6 are concerned with simulations using real-coded GAs.

Our starting point was the scenario underlying the simulations by Arifovic and Gencay (2000). The particular Kareken-Wallace economy in this paper had the following properties: all individuals share a common utility function $U_{i}=c_{i}(t) c_{i}(t+1)$, endowments are $w_{1}=$ $10, w_{2}=4$, and nominal money supplies are $H_{1}=3000, H_{2}=3600$. It can immediately be seen that this leads to a steady state consumption level of $c^{*}=c(t)=c(t+1)=7$ and steady-state savings $s^{*}=s(t)=s(t+1)=3$. Prima facie, it appears unlikely that changes in these economic variables should yield greatly different results (as long as endowments would lead to positive savings). Experimentation with different parameters and alternative utility functions (e.g., logarithmic utility) confirmed this conjecture.

In what follows, we report results on the sensitivity of the model with respect to GA parameters and the number of agents populating the market. Let us start with the effects of varying the GA's parameter settings. From the two parameters of the binary genetic operations, $p_{\text {mut }}$ and $p_{\text {cross }}$, we found the first to be the more interesting one in that variation of $p_{\text {cross }}$ only led to slight variations of the statistical properties. ${ }^{4}$ In a first set of experiments we, therefore, fixed $p_{\text {cross }}$ at 0.6 and also fixed the population size at the level used in Arifovic and Gencay, $N=60$ (i.e., 30 individuals in each generation). ${ }^{5}$ In order to see the effects of variation of $p_{\text {mut }}$, we varied this parameter from 0.005 to 0.05 (with

\footnotetext{
${ }^{4}$ We have redone about half of the cases shown in Tables 1 to 6 with $p_{\text {cross }}$ varying between 0.1 and 0.9 and have found practically no differences in time series characteristics.

${ }^{5}$ It has been suggested by one referee that in the case of few agents one might interpret the GAs as strategies rather than individuals. However, in contrast to the 'market selection' literature, strategies in our GA setting undergo stochastic changes over time. Interpreting the GAs as strategies would, therefore, imply that all adherents of one strategy would also follow the same random processes of mutation and cross-over of this strategy.
} 
Table 1

Variation of tail index estimates for binary coded GAs

\begin{tabular}{|c|c|c|c|c|c|c|c|c|c|}
\hline \multirow[b]{2}{*}{$p_{\text {mut }}$} & \multicolumn{3}{|c|}{ Tail size $2.5 \%$} & \multicolumn{3}{|c|}{ Tail size $5 \%$} & \multicolumn{3}{|c|}{ Tail size $10 \%$} \\
\hline & Min & Median & $\operatorname{Max}$ & Min & Median & Max & Min & Median & Max \\
\hline \multicolumn{10}{|c|}{ Varying mutation probability, constant population size $N=60$} \\
\hline 0.005 & 1.37 & 2.82 & 5.07 & 1.20 & 2.16 & 4.81 & 1.15 & 1.92 & 5.37 \\
\hline 0.010 & 1.93 & 3.36 & 5.06 & 1.35 & 2.81 & 3.81 & 1.34 & 2.21 & 3.06 \\
\hline 0.015 & 2.16 & 3.90 & 5.87 & 1.76 & 3.25 & 4.38 & 1.60 & 2.55 & 3.36 \\
\hline 0.020 & 1.88 & 4.14 & 6.30 & 2.15 & 3.51 & 4.80 & 2.13 & 2.83 & 3.75 \\
\hline 0.025 & 2.60 & 4.45 & 6.87 & 2.60 & 3.81 & 5.47 & 2.31 & 3.04 & 3.72 \\
\hline 0.030 & 3.25 & 4.75 & 7.00 & 3.24 & 4.11 & 5.27 & 2.79 & 3.34 & 3.90 \\
\hline 0.035 & 2.30 & 5.15 & 7.64 & 2.52 & 4.25 & 5.34 & 2.80 & 3.46 & 4.01 \\
\hline 0.040 & 3.50 & 5.13 & 8.38 & 3.23 & 4.42 & 6.64 & 2.85 & 3.61 & 4.40 \\
\hline 0.045 & 3.50 & 5.09 & 7.37 & 3.48 & 4.46 & 6.36 & 3.04 & 3.71 & 4.52 \\
\hline \multirow[t]{2}{*}{0.050} & 3.88 & 5.20 & 7.62 & 3.56 & 4.53 & 5.70 & 3.10 & 3.76 & 5.01 \\
\hline & \multicolumn{3}{|c|}{ Tail size $2.5 \%$} & \multicolumn{3}{|c|}{ Tail size $5 \%$} & \multicolumn{3}{|c|}{ Tail size $10 \%$} \\
\hline$N$ & Min & Median & $\operatorname{Max}$ & Min & Median & $\operatorname{Max}$ & Min & Median & $\operatorname{Max}$ \\
\hline \multicolumn{10}{|c|}{ Constant mutation probability $p_{\text {mut }}=0.01$, varying population size } \\
\hline 20 & 1.73 & 3.29 & 5.43 & 1.38 & 2.64 & 4.67 & 1.30 & 2.26 & 9.17 \\
\hline 100 & 2.76 & 3.77 & 4.56 & 2.12 & 3.17 & 3.85 & 2.02 & 2.49 & 2.79 \\
\hline 200 & 2.29 & 4.19 & 5.67 & 2.26 & 3.54 & 4.64 & 2.16 & 2.68 & 3.16 \\
\hline 1000 & 1.87 & 3.22 & 5.54 & 1.86 & 3.15 & 4.58 & 1.85 & 2.80 & 3.81 \\
\hline 2000 & 1.46 & 2.64 & 4.70 & 1.60 & 2.74 & 4.17 & 1.78 & 2.67 & 3.74 \\
\hline 4000 & 1.19 & 1.94 & 3.78 & 1.52 & 1.92 & 3.53 & 1.56 & 2.20 & 3.21 \\
\hline 10000 & 1.44 & 1.92 & 3.27 & 1.28 & 1.82 & 3.22 & 1.35 & 1.93 & 2.84 \\
\hline
\end{tabular}

Note: Table 1 shows estimates of the parameter $\alpha$ from the asymptotic power-law behavior of large returns: Prob. $\left(\left|r_{t}\right|>x\right) \sim x^{-\alpha}$. We follow the literature in applying a conditional maximum likelihood estimator with a prespecified size of the tail region. To explore the sensitivity of the tail index estimates with respect to the choice of the cut-off, we tried tail regions of 2.5, 5 and 10\%. Empirical estimates usually show a certain tendency of increasing tail indices when the tail size is reduced. For variation of the mutation probability, the minimum, median and maximum over 100 samples with 2000 data points each are shown. For variation of the number of agents, only 25 samples were used due to the increase in computation time with increasing number of GA chromosomes.

increments of 0.005 ) and applied the statistical analyses outlined above to 100 samples each containing 2000 data points (a length of the data series comparable to many empirical records). The 100 samples are taken as non-overlapping windows from a simulation continuing over 205,000 periods where the first 5000 data points have been discarded to account for transient behavior. Minimum, median and maximum of the tail index estimates (for tail sizes of 2.5, 5 and $10 \%$ of the data) are shown in Table 1, while the minimum, median and maximum of the estimates of $d$ are given in the Table 2. Table 3, finally gives the median and range of estimates of the autoregressive parameters from the Dickey Fuller and Augmented Dickey Fuller tests together with the frequencies of one-sided (in parentheses: two-sided) rejection of the unit root null under a 95\% confidence level. Results are quite homogeneous with respect to our three stylized facts in so far as the behavior is most realistic for small values of $p_{\text {mut }}$ around $0.005-0.01$. In this region we have a high percentage of 
Table 2

Variation of index of fractional differentiation for binary coded GAs

\begin{tabular}{|c|c|c|c|c|c|c|c|c|c|}
\hline \multirow[b]{2}{*}{$p_{\text {mut }}$} & \multicolumn{3}{|l|}{ Raw } & \multicolumn{3}{|c|}{ Squared } & \multicolumn{3}{|c|}{ Absolute returns } \\
\hline & Min & Median & Max & Min & Median & Max & Min & Median & $\operatorname{Max}$ \\
\hline \multicolumn{10}{|c|}{ Varying mutation probability, constant population size $N=60$} \\
\hline 0.005 & -1.05 & -0.03 & 0.21 & -0.23 & 0.25 & 0.76 & -0.09 & 0.36 & 0.81 \\
\hline 0.010 & -0.95 & -0.05 & 0.24 & -0.04 & 0.31 & 0.77 & 0.15 & 0.42 & 0.78 \\
\hline 0.015 & -0.61 & -0.12 & 0.29 & 0.02 & 0.34 & 0.81 & 0.11 & 0.43 & 0.88 \\
\hline 0.020 & -0.46 & -0.17 & 0.07 & -0.11 & 0.30 & 0.72 & 0.04 & 0.38 & 0.76 \\
\hline 0.025 & -0.57 & -0.25 & 0.03 & -0.08 & 0.25 & 0.82 & -0.09 & 0.35 & 0.73 \\
\hline 0.030 & -0.58 & -0.30 & 0.07 & -0.11 & 0.23 & 0.51 & -0.07 & 0.29 & 0.60 \\
\hline 0.035 & -0.66 & -0.37 & -0.07 & -0.10 & 0.22 & 0.50 & -0.09 & 0.28 & 0.47 \\
\hline 0.040 & -0.73 & -0.43 & -0.15 & -0.06 & 0.19 & 0.44 & -0.13 & 0.22 & 0.48 \\
\hline 0.045 & -0.76 & -0.46 & -0.14 & -0.12 & 0.19 & 0.49 & -0.07 & 0.22 & 0.50 \\
\hline \multirow[t]{2}{*}{0.050} & -0.84 & -0.51 & -0.18 & -0.13 & 0.15 & 0.47 & -0.10 & 0.18 & 0.52 \\
\hline & \multicolumn{3}{|l|}{ Raw } & \multicolumn{3}{|c|}{ Squared } & \multicolumn{3}{|c|}{ Absolute returns } \\
\hline$N$ & Min & Median & $\operatorname{Max}$ & Min & Median & $\operatorname{Max}$ & Min & Median & $\operatorname{Max}$ \\
\hline \multicolumn{10}{|c|}{ Constant mutation probability $p_{\text {mut }}=0.01$, varying population size } \\
\hline 20 & -0.95 & -0.02 & 0.32 & -0.01 & 0.21 & 0.80 & 0.04 & 0.33 & 0.90 \\
\hline 100 & -0.31 & -0.06 & 0.27 & -0.03 & 0.30 & 0.79 & 0.10 & 0.40 & 0.73 \\
\hline 200 & -0.51 & -0.18 & 0.04 & 0.09 & 0.30 & 0.79 & 0.20 & 0.35 & 0.66 \\
\hline 1000 & -0.76 & -0.56 & -0.32 & -0.12 & 0.13 & 0.34 & 0.01 & 0.16 & 0.34 \\
\hline 2000 & -0.92 & -0.61 & -0.11 & -0.15 & 0.05 & 0.27 & -0.06 & 0.07 & 0.27 \\
\hline 4000 & -0.98 & -0.48 & -0.20 & -0.24 & 0.04 & 0.33 & -0.20 & 0.07 & 0.36 \\
\hline 10000 & -0.85 & -0.40 & -0.11 & -0.23 & 0.01 & 0.13 & -0.12 & 0.04 & 0.22 \\
\hline
\end{tabular}

Note: Table 2 shows estimates of the parameter $d$ for the hyperbolic decay of auto-covariances for variables with long-term dependence: $E\left[x_{t} x_{t-\Delta t}\right] \sim \Delta t^{2 d-1}$. We estimate $d$ via the log periodogram regression technique proposed by Geweke and Porter-Hudak (1983). The underlying data are the same as in Table 1. For variation of the mutation probability, the minimum, median and maximum over 100 samples with 2000 data points each are shown. For variation of the number of agents, only 25 samples were used due to the increase in computation time with increasing number of GA chromosomes.

non-rejection of a unit root in log exchange rates (at least for the ADF test which corrects for short-run dynamics) together with median values of $d$ close to their empirical counterparts. The median tail index estimates might appear somewhat too small, but are still within the range observed with empirical data. Fig. 1 illustrates that returns obtained with this setting of the GA parameters indeed do look very realistic and may be hard to distinguish from real-life records with the naked eye (at least, after, proper adjustment of the scale of the fluctuations). However, when increasing $p_{\text {mut }}$ beyond 0.01 , rejection of a unit root in favor of a root smaller than unity occurs in almost all cases, the fractional differencing parameter for raw returns becomes negative (which is also a signature of mean reversion), and the temporal dependence in squared and absolute returns declines. Finally, the tail index becomes somewhat too high. Fig. 2 shows typical sample paths of log exchange rates for small (0.01) and high (0.05) mutation rates. As can be seen, the latter time series clearly exhibits mean-reverting features (no unit root), while the former shows more pronounced persistence. 
Table 3

Results of unit root tests for binary coded GAs

\begin{tabular}{|c|c|c|c|c|c|c|c|c|}
\hline \multirow[b]{2}{*}{$p_{\text {mut }}$} & \multicolumn{3}{|c|}{ DF test } & \multirow[t]{2}{*}{ Rejections } & \multicolumn{3}{|c|}{ ADF test } & \multirow[t]{2}{*}{ Rejections } \\
\hline & Min & Median & Max & & Min & Median & Max & \\
\hline \multicolumn{9}{|c|}{ Varying mutation probability, constant population size $N=60,100$ runs } \\
\hline 0.005 & 0.15 & 0.97 & 1.00 & $89(85)$ & 0.30 & 0.99 & 1.00 & $54(47)$ \\
\hline 0.010 & 0.47 & 0.97 & 1.00 & $97(95)$ & 0.51 & 0.98 & 1.03 & 64 (59) \\
\hline 0.015 & 0.23 & 0.96 & 0.98 & $100(100)$ & 0.55 & 0.97 & 0.99 & 95 (91) \\
\hline 0.020 & 0.11 & 0.95 & 0.98 & $100(100)$ & 0.42 & 0.96 & 0.99 & 99 (99) \\
\hline 0.025 & 0.61 & 0.93 & 0.97 & $100(100)$ & 0.79 & 0.95 & 0.98 & $100(100)$ \\
\hline 0.030 & 0.68 & 0.92 & 0.96 & $100(100)$ & 0.70 & 0.94 & 0.98 & $100(100)$ \\
\hline 0.035 & 0.20 & 0.91 & 0.96 & $100(100)$ & 0.44 & 0.93 & 0.96 & $100(100)$ \\
\hline 0.040 & 0.49 & 0.91 & 0.95 & $100(100)$ & 0.64 & 0.93 & 0.96 & $100(100)$ \\
\hline 0.045 & 0.27 & 0.90 & 0.95 & $100(100)$ & 0.37 & 0.92 & 0.95 & $100(100)$ \\
\hline \multirow[t]{2}{*}{0.050} & 0.15 & 0.90 & 0.97 & $100(100)$ & 0.33 & 0.91 & 1.01 & 99 (100) \\
\hline & \multicolumn{3}{|c|}{ DF test } & Rejections & \multicolumn{3}{|c|}{ ADF test } & Rejections \\
\hline$N$ & Min & Median & $\operatorname{Max}$ & & Min & Median & Max & \\
\hline \multicolumn{9}{|c|}{ Constant mutation probability, $p_{\text {mut }}=0.01$, varying population size, 25 runs } \\
\hline 20 & 0.14 & 0.95 & 0.99 & $25(25)$ & 0.21 & 0.98 & 1.00 & $14(12)$ \\
\hline 100 & 0.91 & 0.98 & 0.99 & $21(21)$ & 0.94 & 0.99 & 1.00 & $18(18)$ \\
\hline 200 & 0.86 & 0.98 & 0.99 & $25(24)$ & 0.88 & 0.98 & 0.99 & $24(23)$ \\
\hline 1000 & 0.32 & 0.92 & 0.98 & $25(25)$ & 0.41 & 0.91 & 0.97 & $25(25)$ \\
\hline 2000 & 0.16 & 0.78 & 0.98 & $25(25)$ & 0.17 & 0.86 & 0.97 & $25(25)$ \\
\hline 4000 & 0.13 & 0.77 & 0.96 & $25(25)$ & 0.15 & 0.81 & 0.97 & $25(25)$ \\
\hline 10000 & 0.21 & 0.63 & 0.91 & $25(25)$ & 0.26 & 0.56 & 0.93 & $25(25)$ \\
\hline
\end{tabular}

Note: Table 3 shows estimates of the parameter $\rho$ from a regression of the log exchange rate on its lagged value. The columns labeled 'rejection' give the number of cases in which we can reject the unit root null hypothesis $\rho$ $=1$ from the one-sided (two-sided) DF and ADF tests. Again, the underlying data are the ones already analyzed in Tables 1 and 2. Hence, for variation of the mutation probability, the minimum, median and maximum over 100 samples with 2000 data points each are shown. For variation of the number of agents, only 25 samples were used due to the increase in computation time with increasing number of GA chromosomes.

In our second set of experiments, we then varied $\mathrm{N}$ keeping the mutation probability at the value 0.01 .6 Since the simulations become more time-consuming with increasing $N$, we restricted our investigation to 25 samples (i.e. a time series of overall length of 55,000 time steps for each parameter set). Here the changes are even more dramatic. When moving from small $(N=20)$ to very large markets $(N=10,000)$, we get an even larger drop of the autoregressive coefficient in the unit root tests, highly negative $d$ 's for raw returns and a total fading out of volatility clustering (the $d$ 's of squared and absolute returns approaching zero). The tail index decreases and has median values below 2 for the maximum size of the market $(N=10,000)$.

Before turning to explanations, let us look at the pertinent results for real-coded GA's for which we also varied both the set-up of the mutation operator and the number of agents

\footnotetext{
${ }^{6}$ On the request of one referee, we also repeated this chain of experiments with $p_{\text {mut }}=0.005$. Results were virtually identical.
} 
Table 4

Variation of tail index estimates for real-coded GAs

\begin{tabular}{|c|c|c|c|c|c|c|c|c|c|}
\hline \multirow[b]{2}{*}{$p_{\text {mut }}$} & \multicolumn{3}{|c|}{ Tail size $2.5 \%$} & \multicolumn{3}{|c|}{ Tail size $5 \%$} & \multicolumn{3}{|c|}{ Tail size $10 \%$} \\
\hline & Min & Median & Max & Min & Median & Max & Min & Median & Max \\
\hline \multicolumn{10}{|c|}{$\begin{array}{l}\text { Varying mutation probability } p_{\text {mut }} \text {, constant mutation variance } \sigma_{\text {mut }}=0.1 \text { and } \\
\text { population size } N=100\end{array}$} \\
\hline 0.01 & 1.33 & 2.53 & 4.75 & 1.46 & 2.51 & 3.85 & 1.49 & 2.26 & 2.94 \\
\hline 0.02 & 1.75 & 2.57 & 3.93 & 1.62 & 2.33 & 3.47 & 1.54 & 2.08 & 3.18 \\
\hline 0.03 & 0.81 & 3.01 & 6.89 & 0.85 & 2.57 & 3.33 & 0.67 & 2.24 & 2.70 \\
\hline 0.04 & 0.70 & 2.94 & 4.70 & 0.74 & 2.45 & 3.62 & 0.89 & 2.08 & 3.07 \\
\hline 0.05 & 0.96 & 3.23 & 5.02 & 0.73 & 2.68 & 3.62 & 0.85 & 2.33 & 3.09 \\
\hline 0.06 & 0.85 & 2.33 & 3.09 & 0.80 & 2.67 & 4.24 & 1.00 & 2.22 & 3.44 \\
\hline 0.07 & 0.91 & 2.98 & 5.98 & 1.11 & 2.60 & 4.60 & 0.71 & 2.24 & 3.29 \\
\hline 0.08 & 1.03 & 2.93 & 6.18 & 1.04 & 2.62 & 4.65 & 0.86 & 2.14 & 3.51 \\
\hline 0.09 & 0.99 & 2.72 & 6.44 & 1.03 & 2.35 & 4.63 & 0.93 & 2.02 & 3.67 \\
\hline \multirow[t]{2}{*}{0.10} & 1.07 & 2.47 & 6.08 & 0.95 & 2.13 & 3.99 & 0.97 & 1.84 & 3.60 \\
\hline & \multicolumn{3}{|c|}{ Tail size $2.5 \%$} & \multicolumn{3}{|c|}{ Tail size $5 \%$} & \multicolumn{3}{|c|}{ Tail size $10 \%$} \\
\hline$\sigma_{\text {mut }}$ & Min & Median & Max & Min & Median & $\operatorname{Max}$ & Min & Median & $\operatorname{Max}$ \\
\hline
\end{tabular}

Constant mutation probability $p_{\text {mut }}=0.05$, constant population size $N=100$, varying mutation variance

$\begin{array}{llllllllll}0.025 & 1.40 & 3.61 & 5.52 & 1.04 & 3.12 & 4.07 & 1.04 & 2.60 & 3.10 \\ 0.050 & 1.37 & 3.46 & 5.56 & 0.92 & 3.00 & 4.32 & 0.77 & 2.54 & 3.38 \\ 0.075 & 0.67 & 3.37 & 6.34 & 0.65 & 2.95 & 3.97 & 0.59 & 2.47 & 3.32 \\ 0.100 & 0.78 & 3.28 & 5.03 & 0.79 & 2.77 & 4.08 & 1.00 & 2.27 & 3.07 \\ 0.125 & 0.67 & 3.05 & 4.67 & 0.74 & 2.61 & 3.81 & 0.75 & 2.25 & 3.08 \\ 0.150 & 0.70 & 2.91 & 5.15 & 0.89 & 2.57 & 4.41 & 0.71 & 2.14 & 2.99 \\ 0.175 & 0.90 & 2.86 & 5.27 & 0.95 & 2.53 & 3.96 & 0.95 & 2.12 & 3.08 \\ 0.200 & 0.95 & 2.85 & 4.78 & 0.95 & 2.40 & 3.88 & 0.97 & 1.98 & 3.04 \\ & \text { Hill 2.5\% } & & & & & & & \\ \end{array}$

\begin{tabular}{|c|c|c|c|c|c|c|c|c|c|}
\hline \multirow[b]{2}{*}{$N$} & & & \\
\hline & Min & Median & Max & Min & Median & $\operatorname{Max}$ & Min & Median & $\operatorname{Max}$ \\
\hline \multicolumn{10}{|c|}{$\begin{array}{l}\text { Constant mutation probability } p_{\text {mut }}=0.05 \text { and variance } \sigma_{\text {mut }}=0.025 \text {, varying } \\
\text { population size }\end{array}$} \\
\hline 20 & 1.32 & 3.29 & 7.57 & 0.61 & 2.75 & 5.22 & 0.51 & 2.30 & 3.49 \\
\hline 100 & 1.46 & 3.85 & 6.08 & 1.49 & 3.16 & 4.22 & 1.41 & 2.68 & 3.23 \\
\hline 200 & 1.35 & 4.23 & 5.73 & 1.06 & 3.50 & 4.43 & 0.93 & 2.85 & 3.85 \\
\hline 1000 & 0.77 & 4.22 & 8.13 & 0.71 & 3.40 & 6.11 & 0.74 & 3.00 & 4.43 \\
\hline 2000 & 0.85 & 2.65 & 8.16 & 0.68 & 2.39 & 5.97 & 0.62 & 2.08 & 4.52 \\
\hline 4000 & 0.70 & 1.56 & 10.04 & 0.66 & 1.37 & 7.20 & 0.59 & 1.18 & 5.06 \\
\hline 10000 & 0.62 & 1.44 & 8.82 & 0.55 & 1.13 & 6.34 & 0.61 & 0.96 & 5.09 \\
\hline 20000 & 0.52 & 1.49 & 7.99 & 0.62 & 1.14 & 7.24 & 0.58 & 0.86 & 5.04 \\
\hline
\end{tabular}

Note: Table 4 shows estimates of the parameter $\alpha$, now for real-coded GAs. Unlike in Figs. 1-3, we always give the minimum, median and maximum over 100 replications with 2000 data points each even in the case of varying number of agents (real GAs are less demanding in terms of computation time than binary ones).

(Tables 4-6). Again, the parameters of the cross-over operator are kept constant (uniform cross-over as described in Section 3 with a parameter $\gamma=0.2$ was used). The mutation operator now has two parameters, the probability of its activation $p_{\text {mut }}$ and the variance of the Normal mutations, $\sigma_{\text {mut }}$. 
Table 5

Variation of index of fractional differentiation for real-coded GAs

\begin{tabular}{|c|c|c|c|c|c|c|c|c|c|}
\hline \multirow[b]{2}{*}{$p_{\text {mut }}$} & \multicolumn{3}{|l|}{ Raw } & \multicolumn{3}{|c|}{ Squared } & \multicolumn{3}{|c|}{ Absolute returns } \\
\hline & Min & Median & Max & Min & Median & Max & Min & Median & $\operatorname{Max}$ \\
\hline \multicolumn{10}{|c|}{$\begin{array}{l}\text { Varying mutation probability } p_{\text {mut }} \text {, constant mutation variance } \sigma_{\text {mut }}=0.1 \text { and } \\
\text { population size } N=100\end{array}$} \\
\hline 0.01 & -1.06 & -0.39 & 0.24 & -0.28 & 0.09 & 0.41 & -0.22 & 0.17 & 0.52 \\
\hline 0.02 & -0.79 & -0.05 & 0.25 & -0.16 & 0.14 & 0.43 & -0.22 & 0.24 & 0.55 \\
\hline 0.03 & -0.44 & -0.04 & 0.32 & -0.14 & 0.13 & 0.90 & -0.08 & 0.22 & 1.11 \\
\hline 0.04 & -0.52 & -0.06 & 0.22 & -0.08 & 0.28 & 1.04 & -0.10 & 0.40 & 1.07 \\
\hline 0.05 & -0.44 & -0.03 & 0.25 & -0.11 & 0.25 & 1.17 & -0.01 & 0.35 & 1.16 \\
\hline 0.06 & -0.48 & -0.08 & 0.29 & -0.02 & 0.28 & 1.09 & -0.03 & 0.40 & 1.23 \\
\hline 0.07 & -0.52 & -0.08 & 0.21 & -0.11 & 0.30 & 0.87 & -0.06 & 0.47 & 1.03 \\
\hline 0.08 & -0.43 & -0.07 & 0.22 & -0.03 & 0.34 & 0.93 & -0.04 & 0.48 & 0.99 \\
\hline 0.09 & -0.52 & -0.11 & 0.26 & -0.01 & 0.34 & 0.89 & -0.05 & 0.50 & 1.32 \\
\hline \multirow[t]{2}{*}{0.10} & -0.48 & -0.13 & 0.17 & 0.05 & 0.35 & 1.00 & 0.06 & 0.53 & 0.92 \\
\hline & \multicolumn{3}{|l|}{ Raw } & \multicolumn{3}{|c|}{ Squared } & \multicolumn{3}{|c|}{ Absolute returns } \\
\hline$\sigma_{\text {mut }}$ & Min & Median & Max & Min & Median & $\operatorname{Max}$ & Min & Median & $\operatorname{Max}$ \\
\hline
\end{tabular}

Constant mutation probability $p_{\text {mut }}=0.05$, constant population size $N=100$, varying mutation variance

\begin{tabular}{|c|c|c|c|c|c|c|c|c|c|}
\hline 0.025 & -0.30 & -0.01 & 0.34 & -0.22 & 0.16 & 0.98 & -0.10 & 0.24 & 0.98 \\
\hline 0.050 & -0.48 & -0.07 & 0.21 & -0.40 & 0.19 & 1.03 & -0.06 & 0.26 & 1.01 \\
\hline 0.075 & -0.52 & -0.04 & 0.30 & -0.03 & 0.22 & 0.90 & -0.04 & 0.29 & 1.14 \\
\hline 0.100 & -0.39 & -0.05 & 0.27 & -0.09 & 0.30 & 1.14 & -0.07 & 0.45 & 1.08 \\
\hline 0.125 & -0.56 & -0.06 & 0.31 & -0.08 & 0.31 & 0.94 & -0.18 & 0.46 & 1.03 \\
\hline 0.150 & -0.42 & -0.06 & 0.17 & -0.10 & 0.31 & 1.05 & 0.01 & 0.43 & 1.10 \\
\hline 0.175 & -0.50 & -0.07 & 0.25 & 0.02 & 0.34 & 1.13 & -0.06 & 0.49 & 1.07 \\
\hline \multirow[t]{3}{*}{0.200} & -0.46 & -0.09 & 0.19 & -0.21 & 0.35 & 1.00 & -0.02 & 0.50 & 1.00 \\
\hline & \multicolumn{3}{|l|}{ Raw } & \multicolumn{3}{|c|}{ Squared } & \multicolumn{3}{|c|}{ Absolute returns } \\
\hline & Min & Median & Max & Min & Media & Max & Min & Median & $\operatorname{Max}$ \\
\hline
\end{tabular}

Constant mutation probability $p_{\text {mut }}=0.05$ and variance $\sigma_{\text {mut }}=0.025$, varying population size

$\begin{array}{rrrrrrrrrr}20 & -0.48 & -0.02 & 0.46 & -0.10 & 0.21 & 0.74 & -0.07 & 0.29 & 0.89 \\ 100 & -0.37 & 0.00 & 0.21 & -0.16 & 0.13 & 0.65 & -0.16 & 0.20 & 0.71 \\ 200 & -0.60 & 0.01 & 0.30 & -0.16 & 0.12 & 0.73 & -0.18 & 0.15 & 0.81 \\ 1000 & -0.76 & -0.02 & 0.40 & -0.27 & 0.13 & 0.71 & -0.26 & 0.22 & 1.05 \\ 2000 & -0.66 & 0.03 & 0.39 & -0.31 & 0.11 & 0.85 & -0.33 & 0.28 & 1.02 \\ 4000 & -0.79 & -0.15 & 0.39 & -0.31 & 0.10 & 0.39 & -0.34 & 0.29 & 0.98 \\ 10000 & -0.72 & -0.28 & 0.45 & -0.30 & 0.13 & 0.86 & -0.28 & 0.37 & 0.96 \\ 20000 & -0.75 & -0.36 & 0.47 & -0.13 & 0.10 & 0.86 & -0.03 & 0.34 & 1.19\end{array}$

Note: Table 5 shows estimates of the parameter $d$, now for real-coded GAs. The data are the same as in Table 4.

The upper and middle part of Tables 4-6 exhibit the effects of systematic variation of $p_{\text {mut }}$ and $\sigma_{\text {mut }}$, the bottom part shows the effects of varying population size. Again, 100 samples of 2000 data points each have been used. Since markets with real-coded GA's need less computation time, we were able to use 100 subsamples when assessing the effects of market size and could also use a somewhat larger maximum size of $N=20,000$. 
Table 6

Results of unit root tests for real-coded GAs

\begin{tabular}{|c|c|c|c|c|c|c|c|c|}
\hline \multirow[b]{2}{*}{$p_{\text {mut }}$} & \multicolumn{3}{|l|}{ DF test } & \multirow[t]{2}{*}{ Rejections } & \multicolumn{3}{|c|}{ ADF test } & \multirow[t]{2}{*}{ Rejections } \\
\hline & Min & Median & Max & & Min & Median & Max & \\
\hline \multicolumn{9}{|c|}{$\begin{array}{l}\text { Varying mutation probability } p_{\text {mut }} \text {, constant mutation variance } \sigma_{\text {mut }}=0.1 \text { and } \\
\text { population size } N=100,100 \text { runs }\end{array}$} \\
\hline 0.01 & 0.29 & 0.90 & 1.00 & $74(70)$ & 0.42 & 0.94 & 1.00 & $59(61)$ \\
\hline 0.02 & 0.83 & 0.99 & 1.00 & 54 (49) & 0.87 & 0.99 & 1.00 & $54(49)$ \\
\hline 0.03 & -0.00 & 0.99 & 1.00 & $50(47)$ & 0.01 & 0.99 & 1.00 & $35(34)$ \\
\hline 0.04 & -0.00 & 0.99 & 1.00 & 49 (42) & 0.06 & 0.99 & 1.00 & $34(30)$ \\
\hline 0.05 & 0.01 & 0.99 & 1.01 & $46(45)$ & 0.26 & 0.99 & 1.00 & $39(37)$ \\
\hline 0.06 & -0.00 & 0.99 & 1.00 & $55(45)$ & -0.00 & 0.99 & 1.00 & $40(34)$ \\
\hline 0.07 & 0.00 & 0.99 & 1.00 & $56(47)$ & 0.00 & 0.99 & 1.01 & $40(36)$ \\
\hline 0.08 & 0.00 & 0.99 & 1.00 & $58(51)$ & 0.01 & 0.99 & 1.00 & $46(40)$ \\
\hline 0.09 & -0.00 & 0.99 & 1.00 & $53(48)$ & -0.00 & 0.99 & 1.00 & $48(41)$ \\
\hline \multirow[t]{2}{*}{0.10} & -0.00 & 0.98 & 1.01 & $68(66)$ & -0.00 & 0.98 & 1.01 & $68(63)$ \\
\hline & \multicolumn{3}{|l|}{ DF test } & Rejections & \multicolumn{3}{|c|}{ ADF test } & Rejections \\
\hline$\sigma_{\text {mut }}$ & Min & Median & Max & & Min & Median & Max & \\
\hline
\end{tabular}

Constant mutation probability $p_{\text {mut }}=0.05$, constant population size $N=100$, varying mutation variance, 100 runs

\begin{tabular}{|c|c|c|c|c|c|c|c|c|}
\hline 0.025 & -0.00 & 1.00 & 1.00 & $36(30)$ & -0.00 & 1.00 & 1.00 & $25(24)$ \\
\hline 0.050 & 0.03 & 0.99 & 1.00 & $43(32)$ & 0.19 & 1.00 & 1.00 & $26(21)$ \\
\hline 0.075 & 0.01 & 0.99 & 1.00 & $51(40)$ & 0.01 & 0.99 & 1.00 & $34(33)$ \\
\hline 0.100 & -0.00 & 0.99 & 1.00 & $69(60)$ & 0.31 & 0.99 & 1.00 & $54(48)$ \\
\hline 0.125 & -0.00 & 0.99 & 1.00 & $68(65)$ & -0.00 & 0.99 & 1.03 & $55(53)$ \\
\hline 0.150 & -0.00 & 0.99 & 1.00 & $64(58)$ & -0.00 & 0.99 & 1.00 & $46(39)$ \\
\hline 0.175 & -0.00 & 0.99 & 1.00 & $76(66)$ & 0.12 & 0.99 & 1.10 & $50(48)$ \\
\hline \multirow[t]{3}{*}{0.200} & -0.00 & 0.98 & 1.00 & 72 (69) & 0.02 & 0.99 & 7.71 & $60(57)$ \\
\hline & \multicolumn{3}{|l|}{ DF test } & Rejections & \multicolumn{3}{|c|}{ ADF test } & Rejections \\
\hline & Min & Median & Max & & Min & Median & Max & \\
\hline
\end{tabular}

\begin{tabular}{|c|c|c|c|c|c|c|c|c|}
\hline \multicolumn{9}{|c|}{$\begin{array}{l}\text { Constant mutation probability } p_{\text {mut }}=0.05 \text { and variance } \sigma_{\text {mut }}=0.025 \text {, varying } \\
\text { population size, } 100 \text { runs }\end{array}$} \\
\hline 20 & 0.77 & 0.98 & 1.00 & 85 (79) & 0.88 & 0.99 & 1.00 & $21(17)$ \\
\hline 100 & -0.00 & 1.00 & 1.00 & $29(24)$ & 0.00 & 1.00 & 1.00 & $19(15)$ \\
\hline 200 & 0.99 & 1.00 & 1.00 & $2(4)$ & 0.99 & 1.00 & 1.00 & $4(4)$ \\
\hline 1000 & -0.00 & 1.00 & 1.76 & $30(38)$ & -0.00 & 1.00 & 24.74 & $31(33)$ \\
\hline 2000 & -0.00 & 1.00 & 1.66 & $24(33)$ & -0.00 & 1.00 & 6.11 & 27 (29) \\
\hline 4000 & -0.00 & 1.00 & 1.06 & $37(50)$ & -0.00 & 1.00 & 1.02 & $35(43)$ \\
\hline 10000 & 0.01 & 1.00 & 1.52 & $45(63)$ & 0.01 & 1.00 & 12.52 & $44(52)$ \\
\hline 20000 & 0.12 & 1.00 & 1.03 & 43 (54) & 0.12 & 1.00 & 1.01 & 42 (49) \\
\hline
\end{tabular}

Note: Table 6 shows results from unit root tests, now for real-coded GAs. The underlying data are the same as in Tables 4 and 5 .

In broad harmony with the binary-coded case, realistic properties are obtained with small mutation probability and small variance of mutation. Nevertheless, certain differences are observed between the binary and real-coded cases: in particular, the DF and ADF tests are still unable to reject the unit root hypothesis in the majority of cases even with a 

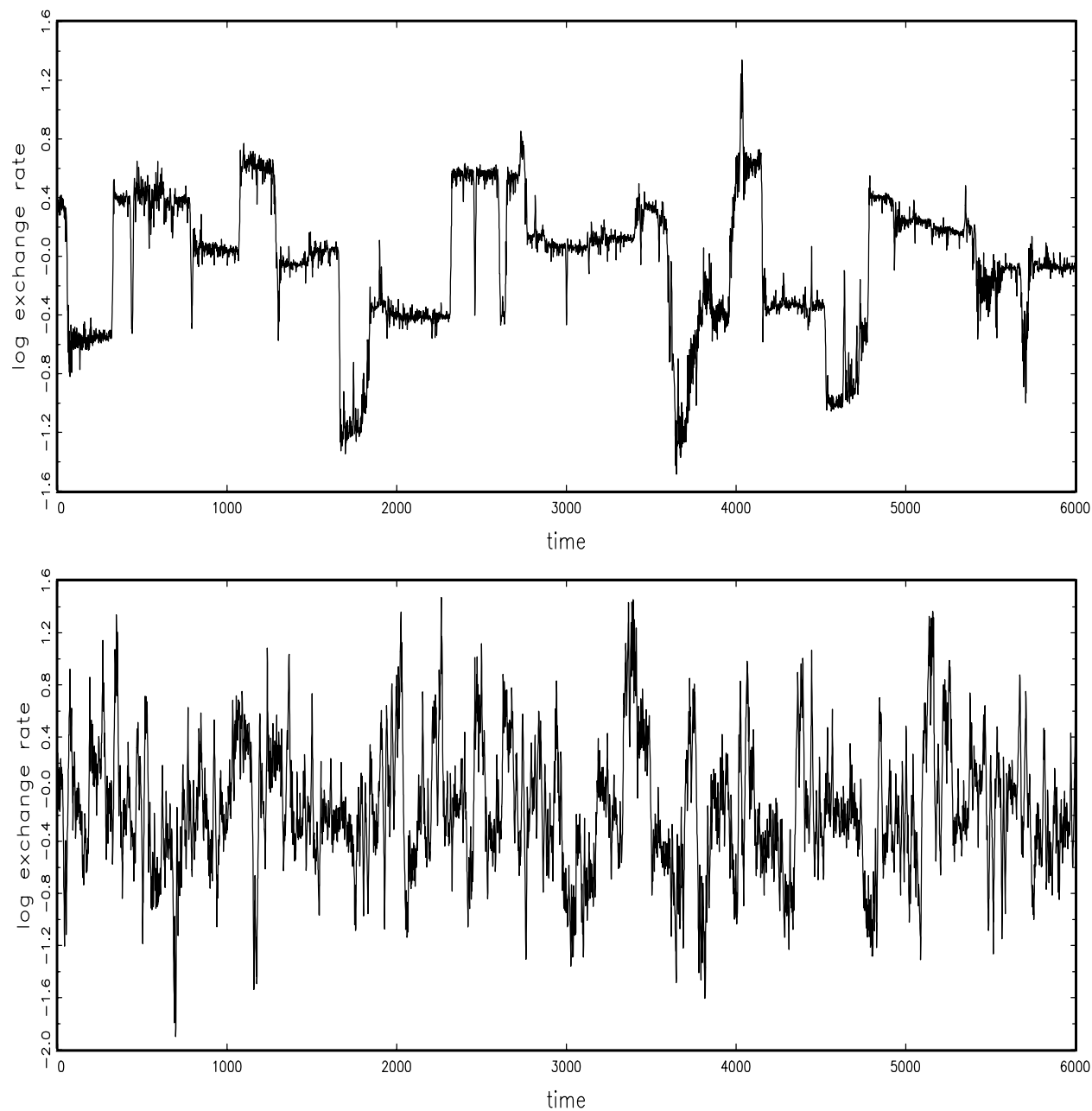

Fig. 2. Log exchange rates from simulated economies with $p_{\text {mut }}=0.01$ (upper panel) and $p_{\text {mut }}=0.05$ (lower panel). The population consists of 100 binary-coded GAs in both cases. Although the simulated exchange rate revolution does not appear fully realistic, for parts of the upper time series the DF and ADF tests are unable to reject the unit root null. The simulation in the lower panel has more easily recognizable mean-reverting features.

relatively large mutation rate and a large number of traders. On the other hand, mean reverting tendencies are clearly observable in the estimates of the fractional differencing parameter for raw returns in these cases. It might be that the evolution of the system is simply very slow so that in many periods the slight variations in the exchange rate go through as a unit root process. In contrast to the binary case, in the real-coded one the two-sided test sometimes yields more rejections than the one-sided test. The additional right-hand rejections may be related to the sudden bursts of activity visible in the upper 

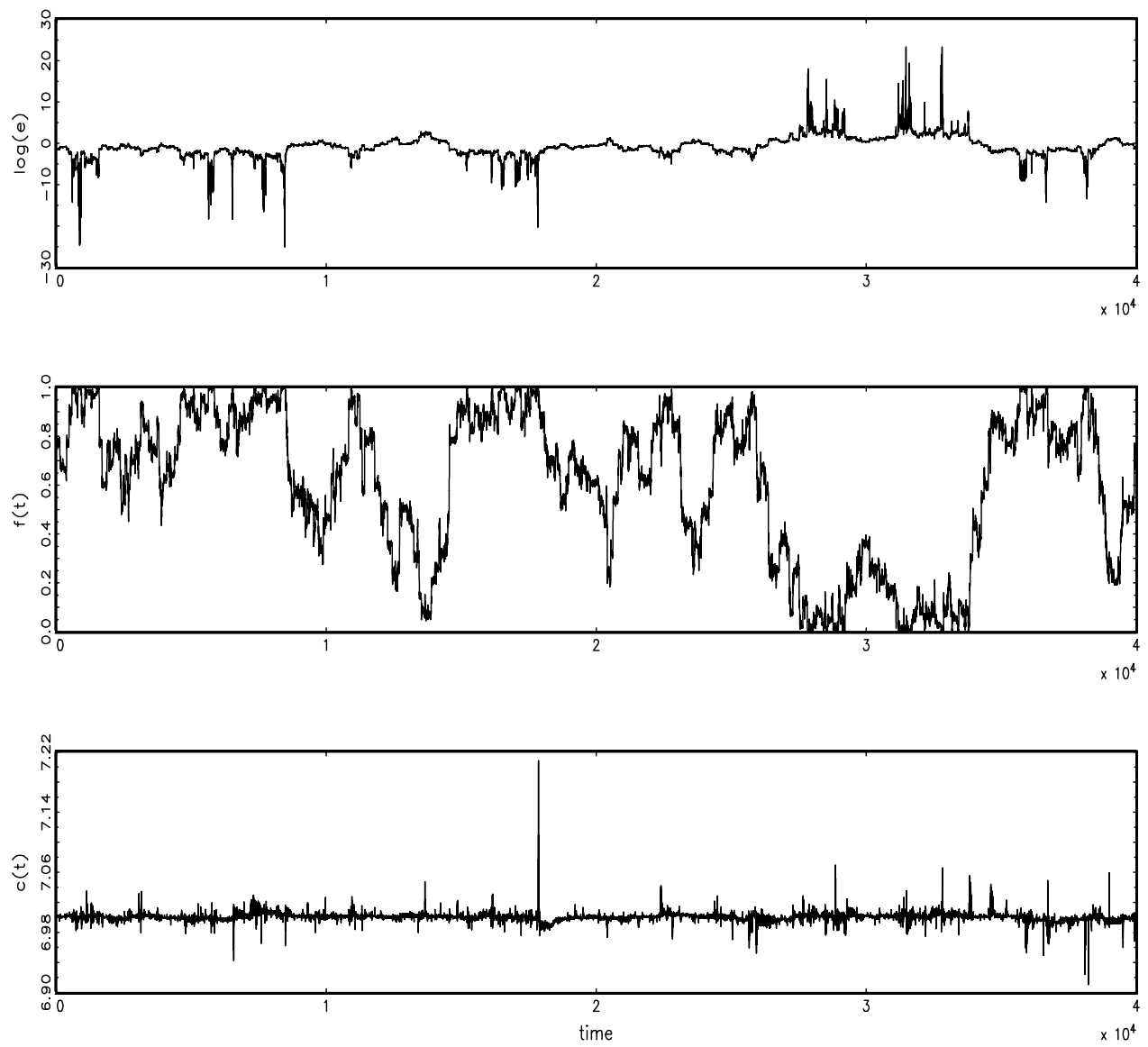

Fig. 3. Log exchange rate (top), average portfolio fraction of home currency (middle), and average first-period consumption (bottom) for a real-coded GA population of 200 agents. For economic parameters, see main text. GA parameters are: $p_{\text {mut }}=0.05, \sigma_{\text {mut }}=0.025$ and $\gamma=0.2$. The dynamics seems to be characterized by unsystematic changes of the portfolio composition which lead to exchange rate fluctuations, but leave average consumption choices almost unaffected.

part of Fig. 3. Overall, the much higher rate of rejection of the unit root hypothesis in the binary case might be due to the higher degree of stochasticity inherited from thirty instances of mutation (for every bit) instead of two instances only in the real-coded case. Some reflection, in fact, reveals that the values of the mutation probabilities can not be directly compared between the binary coded and real-coded GAs. For example, a $p_{\text {mut }}$ $=0.033$ (per bit) for binary coded GAs implies that with chromosomes of thirty bits, almost every individual will undergo some mutation of its genetic material. This amounts to a much higher mutation rate within the population with $p_{\text {mut }}=0.01$ in the binary case than with 0.05 for real-coded bits. Except for non-rejection of the unit root hypothesis, the pattern of results is, in fact, almost the same as with binary GAs when increasing $N$. 

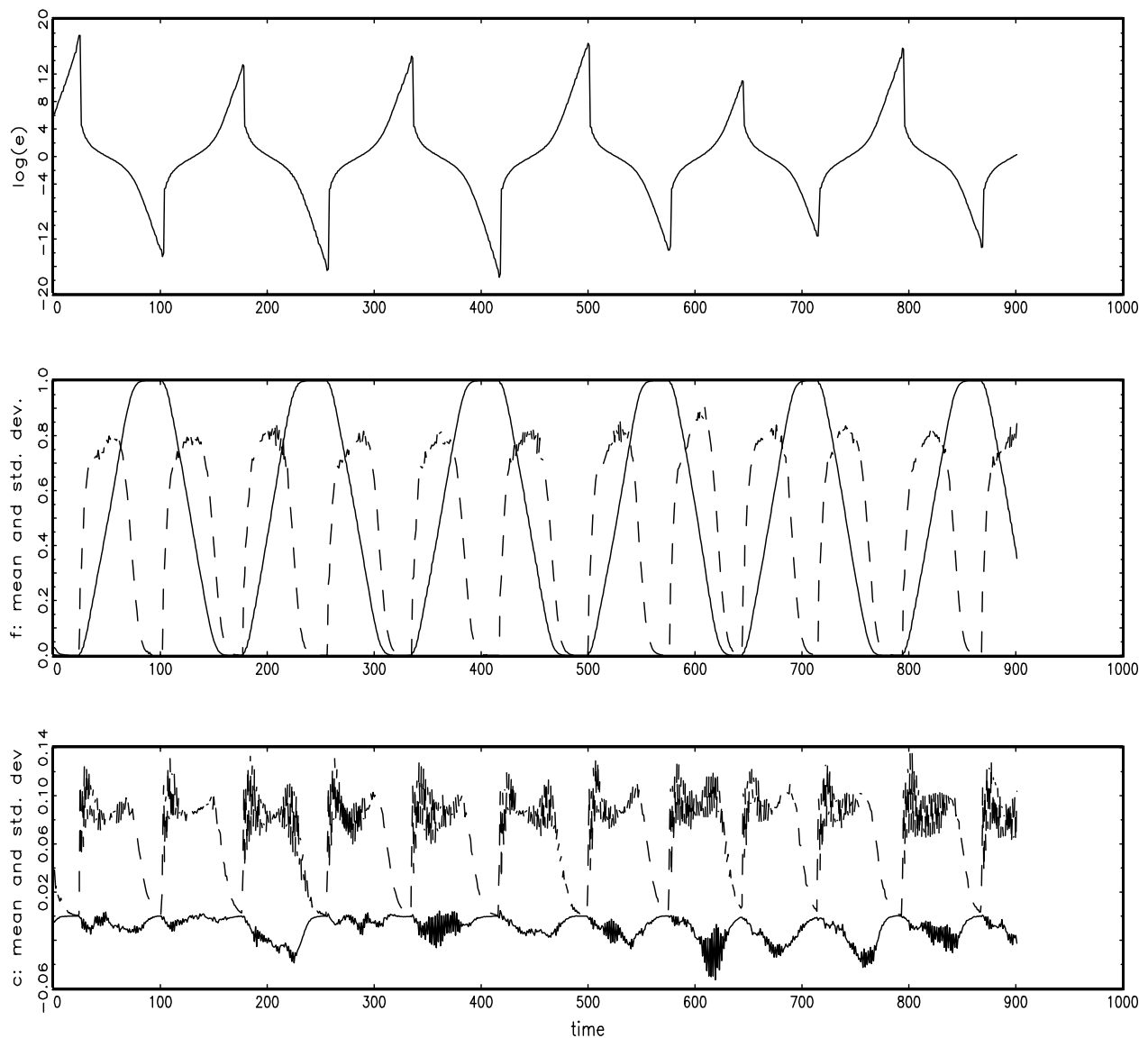

Fig. 4. Log exchange rate (top), average portfolio fraction of home currency (middle), and average first-period consumption (bottom) for a real-coded GA population of 20,000 agents. For economic parameters, see main text. GA parameters are: $p_{\text {mut }}=0.3, \sigma_{\text {mut }}=0.025$ and $\gamma=0.2$. The middle and bottom panels show both the mean (solid lines) and standard deviations (broken lines) of the distribution of the choice variables within the population. In order to lodge the mean and standard deviation in the bottom panel, we have subtracted the steady state value $c^{*}$ $=7$, reduced the standard deviation of $c_{i}(t)$ by one-half and magnified the standard deviation of $f_{i}(t)$ by a factor 10 . The higher $p_{\text {mut }}$ compared to Fig. 3 mainly serves to decrease the amplitude of the cycle and enhance convergence to regular cycles.

Looking at the resulting time series, we find in both the real-coded and binary coded case a tendency towards persistent and very regular cycles when increasing the number of agents (cf. Figs. 3 and 4).

Besides this similarity in the results from both GA variants (and a number of alternative implementations of various operators) our experiments also show that we do not need all of the typical elements of the GA to arrive at these results. Essentially, the structure of the artificial economy remains unchanged if we dispense with both the selection and cross-over operators. Mutation and election alone are capable of producing these patterns, but they 
are also crucial for their emergence (although selection and cross-over tend to speed up convergence to regular oscillations).

\section{Interpretation of results}

What is the reason for this fading out of realistic time series properties with increasing mutation probability and increasing number of agents? It is probably not too difficult to answer the first part of the question: a high mutation probability introduces a certain tendency of mean-reversion of the choice parameters which is reflected in similar mean reversion of the exchange rate. For the sake of illustration, imagine a model with a mutation probability equal to 1 in the case of binary coded GAs (corresponding to a $p_{\text {mut }}=1$ together with a high variance of mutation in the real-coded case). This would lead to a stationary random distribution of agents' strategies. All deviations from the average would be corrected by the new random choice of the population in the next period and, hence, one gets a tendency of return to the mean values of the distribution of $c_{i}(t)$ and $f_{i}(t)$. Since these choice variables determine prices and the exchange rate, mean-reversion would also carry over to these variables as well. The higher the mutation rate, the higher the influence of this tendency. Higher $p_{\text {mut }}$, therefore, leads to less persistence in exchange rates so that the exchange rate dynamics becomes stationary and unit roots can be rejected. This suspected change in the appearance of the time series is already well recognizable when comparing binary coded GAs with $p_{\text {mut }}=0.01$ and 0.05 , respectively (cf. Fig. 2). While the former series (or parts of it) might be accepted as a random walk by the DF and ADF tests, the second surely will not. As a conclusion, we infer from these considerations that random experimentation with strategies has to be limited in order to get realistic appearance of the time series.

As to the number of agents, a glance at the time series resulting with different sizes of the market reveals some perplexing patterns (Figs. 3 and 4). What looks extremely unsystematic with a small market $(N=200)$, becomes much more regular when the market becomes larger and eventually evolves into an almost perfectly regular oscillatory motion of some 'macroscopic' variables (in particular, the exchange rate and the average fraction of domestic currency in the portfolio). ${ }^{7}$ On a close inspection, the short spikes in the exchange rate fluctuations in Fig. 3 are very similar to one half-cycle in Fig. 4. Such a regular pattern is puzzling at first view as it is the result of the evolving decisions of a very large ensemble of autonomous artificial agents. Keeping in mind that the quantity displayed in the middle part of Figs. 3 and 4 is a population average what these oscillations show is a systematic shift of the whole distribution of this variable within an heterogeneous ensemble of agents. To our knowledge nothing of a similar type of self-organizing patterns is known in multi-agent systems with GA learning in economics or other fields. A certain clue to the underlying mechanisms can be obtained through analysis of what happens in the case of a large economy (i.e., with the number of agents going to infinity). Noting that GAs are

\footnotetext{
${ }^{7}$ We have chosen a higher mutation probability compared to our benchmark case in Fig. 4 since it both leads to an increase in the frequency of the oscillations and provides faster convergence to almost perfectly regular patterns. However, the trend to emerging regular oscillations is also clearly visible in other simulations with either real-coded or binary GAs.
} 
an adaptive adjustment scheme that drives the actual average behavior of the population towards the momentary optimum of the choice variables, the large economy case might be described via the resulting deterministic mean value dynamics of the choice variables. Unfortunately, the present dynamics is too complicated to derive explicit dynamics laws for the large economy limit. ${ }^{8}$ However, some heuristic considerations will reveal most of the important elements of our dynamics.

As an adaptive adjustment scheme, the genetic algorithm has at its intrinsic benchmark those values $c_{i}{ }^{*}(t)$ and $f_{i}^{*}(t)$ which would have been optimal choices for the population at time $t$ which inherits its genetic material to the generation born at time $t+2$.

It is easy to see that for our utility function $U_{i}=c_{i}(t) c_{i}(t+1)$ optimal behavior of individual $i$ at time $t$ would have been:

$$
\begin{aligned}
c_{i}^{*}(t) & =\frac{1}{2}\left(w_{1}+\frac{w_{2}}{f_{i}(t) p_{1}(t) / p_{1}(t+1)+\left(1-f_{i}(t)\right) p_{2}(t) / p_{2}(t+1)}\right), \\
f_{i}^{*}(t) & =\left\{\begin{array}{ccc}
1 & > \\
\text { indeterminate } & \text { if } \frac{p_{1}(t)}{p_{1}(t+1)} & =\frac{p_{2}(t)}{p_{2}(t+1)} . \\
0 & & <
\end{array}\right.
\end{aligned}
$$

Via prices at period $t+1$, the optimal behavior of generation $t$ also depends on the decisions of the next generation. The election operator guarantees that the resulting new individuals emerging from selection, cross-over and mutation are at least as good as their parents. For the portfolio component, this clearly implies $f_{i}(t+2) \in\left[f_{i}(t), f_{i}^{*}(t)\right]$ while consumption might also overshoot its target, $c_{i}{ }^{*}(t)$ as long as the resulting new parameter set provides at least the utility level enjoyed by the parent individuals. Since in any out-of-equilibrium situation, the goal value for the fraction of domestic assets will be the same, 0 or 1 , for all members of the population, in the large economy limit, the motion of the mean value $\overline{f(t)}$ will follow a deterministic path towards these values as long as the pay-off differential from holding domestic or foreign currency does not change.

To see the source of the regular fluctuations, consider a stationary situation with homogeneous choice variables $c_{i}(t)=c_{i}(t-1)=c^{*}$ and $f_{i}(t)=f_{i}(t-1)=f_{0}$, where $f_{0}$ might be any admissible value between 0 and 1 . Arifovic (1996) has already emphasized that any such equilibrium of this GA economy is evolutionary unstable since any local disturbance (mutation) will be magnified by the ensuing adjustments of the remaining members of the population. For the sake of the argument, assume that only one individual undergoes a mutation when the generation $t+1$ emerges from the genetic operations on generation $t-1$. Assume furthermore that this mutation amounts to an increase of the fraction of domestic money in the portfolio of this agent, while its consumption remains unchanged at the initial equilibrium level. Since this new strategy will have the same utility as its parents (because returns from holding either currency are initially identical), the election operator will allow this offspring to replace one of its parents. However, the presence of this mutant suffices

\footnotetext{
${ }^{8}$ Available analytical approaches to genetic algorithm dynamics consider simpler examples and are not applicable to the present model (e.g., Prügel-Bennett and Shapiro, 1994, or Srinivas and Patnaik, 1996).
} 
to change the structure of returns for agents of generation t: instead of equal returns, they experience a higher pay-off from holding domestic money:

$$
R_{1}(t)=\frac{\sum_{i} f_{i}(t+1)\left(w_{1}-c^{*}\right)}{\sum_{i} f_{i}(t)\left(w_{1}-c^{*}\right)}>R_{2}(t)=\frac{\sum_{i}\left(1-f_{i}(t+1)\right)\left(w_{1}-c^{*}\right)}{\sum_{i}\left(1-f_{i}(t)\right)\left(w_{1}-c^{*}\right)} .
$$

Although the differential might be very small, it suffices to make $f^{*}=1$ the dominant strategy. Hence, the random change from generation $t-1$ to generation $t+1$ induces a systematic shift into the same direction when generation $t$ inherits its genetic material to generation $t+2$. Does it also lead to changes in the consumption behavior of generation $t+2$ ? Changes in the momentary optimal consumption level occur if the denominator in Eq. (4.a) deviates from one. With consumption still equal to its steady state level at generations $t$ and $t+1$, this denominator amounts to $f_{i}(t) \overline{f(t+1)} / \overline{f(t)}+\left(1-f_{i}(t)\right)(1-$ $\overline{f(t+1)}) /(1-\overline{f(t)})$. Given our assumption, $\overline{f(t+1)}>\overline{f(t)}$, it is easy to see that the denominator is $>(<) 1$ for individuals with $f_{i}(t)>(<) \overline{f(t)}$. It would, therefore, be optimal for the former to reduce first-period consumption, while the later would find it advantageous to increase it. However, since we have also assumed that all individuals share the same choice variable $f_{i}(t)=f_{0}=\overline{f(t)}$ at time $t$, the former consumption level $c^{*}$ would still be optimal for all generation $t$ members as long as their portfolio choice remains unaffected by the genetic operations, so that isolated changes of $c_{i}(t)$ would not survive the election operator test. Note that these considerations apply only in the case of isolated genetic changes of either $f_{i}(t)$ or $c_{i}(t)$. Often both variables will be affected by the genetic processes. To see more generally, what kind of arbitrary combined genetic changes would survive in our scenario, we can take stock of the traditional concept of indifference curves. Accepting only offspring who are at least as fit as their parents, the election operator only allows those to enter the population whose choice variables positions them on the same indifference curve like their parents or a higher indifference curve. Consider the utility obtained by parent individual $i$ :

$$
\begin{aligned}
& U_{i}(t)=c_{i, 1}(t)\left(w_{2}+\left(w_{1}-c_{i, 1}(t)\right) \rho_{i}(t)\right), \\
& \text { with } \rho_{i}(t)=f_{i}(t) R_{1}(t)+\left(1-f_{i}(t)\right) R_{2}(t) .
\end{aligned}
$$

The slope of an indifference curve is given by:

$$
\left.\frac{\mathrm{d} c_{1, i}(t)}{\mathrm{d} f_{i}(t)}\right|_{U=\mathrm{const} .}=\frac{-c_{i, 1}(t)\left(w_{1}-c_{i, 1}(t)\right)\left(R_{1}(t)-R_{2}(t)\right)}{w_{2}+\left(w_{1}-2 c_{i, 1}(t)\right) \rho_{i}(t)} .
$$

For $R_{1}(t)>R_{2}(t)$ this gives:

$$
\left.\frac{\mathrm{d} c_{i, 1}(t)}{\mathrm{d} f_{i}(t)}\right|_{U=\text { const. }}<_{0}^{>} \text {if } \quad c_{i, 1}(t) \underset{<}{>} c^{*}\left(f_{i}(t)\right)=0.5\left(w_{1}+w_{2} / \rho_{i}(t)\right) .
$$

This yields the parabolic shape of the indifference curves exhibited in Fig. 5. In the case of $R_{1}(t)<R_{2}(t)$, the inequalities in Eq. (8) are reversed and the indifference curve parabolas have the opposite orientation. In the present case, $R_{1}(t)>R_{2}(t)$, it can be inferred from Eq. (7), that higher utility can only be achieved if a higher fraction of domestic assets is chosen. If, however, this necessary condition for an improvement is met, a certain range of higher or lower consumption levels would be accepted by the election operator. As can 


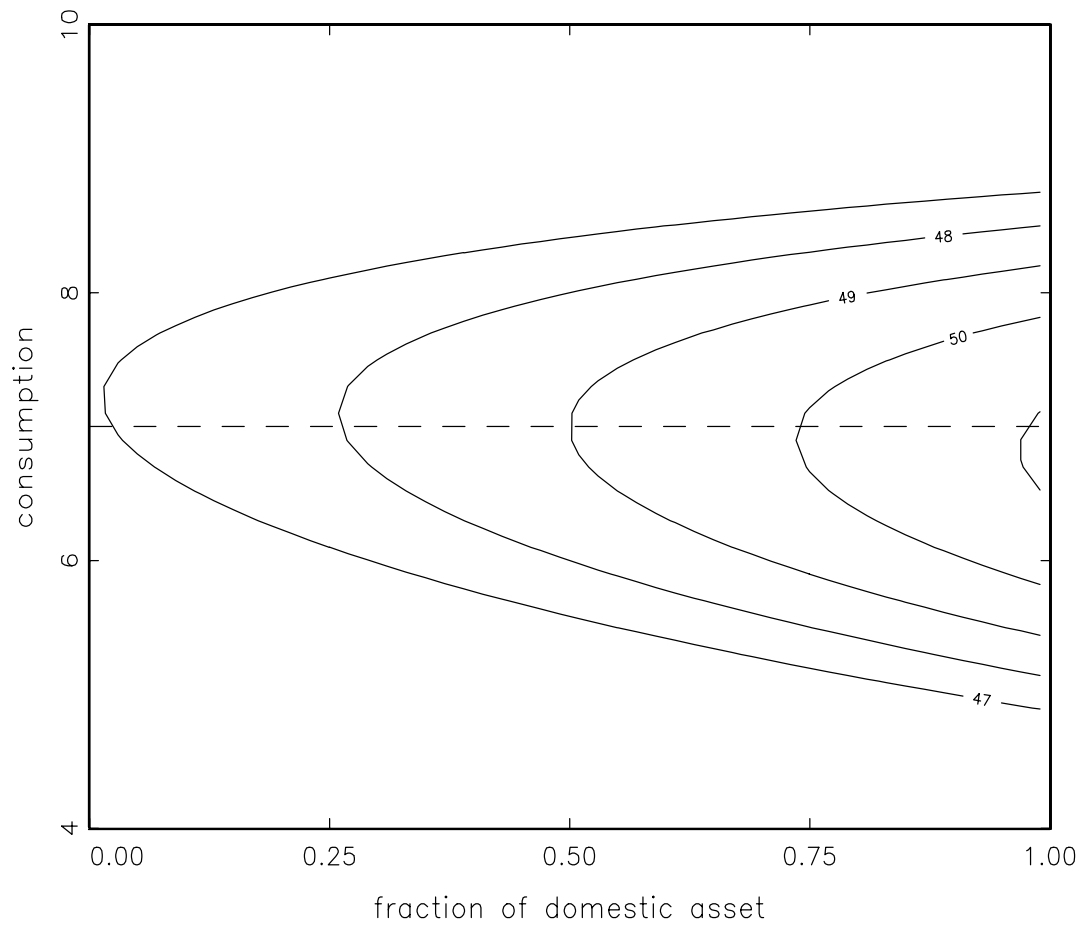

Fig. 5. Indifference curves. The underlying utility function is $U=c(t) c(t+1)$, endowments are $w_{1}=10, w_{2}=4$. For this illustration it has been assumed that all agents have chosen their first-period consumption level equal to its steady state value, $c^{*}=7$, and that the fraction of domestic money in the portfolios of generations $t$ and $t+1$ has mean values $\overline{f(t+1)}=0.55$ and $\overline{f(t)}=0.5$.

be seen from Fig. 5, in the above situation in which $\overline{f(t+1)}$ slightly exceeds $\overline{f(t)}$, the spectrum of utility improving changes is slightly asymmetric with respect to consumption. Assuming that all initial consumption levels are close to $c^{*}$, the pay-offs can be reduced to: $R_{1}(t)=\overline{f(t+1)} / \overline{f(t)}, R_{2}(t)=(1-\overline{f(t+1)}) /(1-\overline{f(t)})$. According to the arguments given above, individuals with a below (above) average fraction of domestic money would, then, have $c^{*}\left(f_{i}(t)\right)>(<) c^{*}=0.5\left(w_{1}+w_{2}\right)$. The expected direction of combined changes of $c_{i}$ and $f_{i}$ would, therefore, depend on the individual's position within the distribution of the $f_{i}$ 's. For roughly half of the population on average somewhat higher consumption levels would pass the election operator, while for the other half of its members, the genetic operations would slightly favor a reduction in consumption. With a symmetric distribution of the $f_{i}$ 's, the expected macroscopic effect of induced changes of $c_{i}$ would be close to zero. With an asymmetric distribution, skewness would somewhat favor one or the other direction of changes, but since this is a third-order effect, one might expect it to be negligible. This conjecture is supported by our simulations which show a high level of stochasticity in the development of $c_{i}(t)$ over time even with a very large population. What can be observed, however, is that an increase of the number of agents leads to a reduction of the size of fluctuations of $c_{i}(t)$, cf. Figs. 3 and 4. 
With only small changes of $c_{i}(t)$, the systematic changes of the portfolio composition will dominate the dynamics. The attraction towards the extreme solutions will, then, be self-reinforcing leading to an ever increasing fraction of domestic assets in the upward part of the cycle. Since every new round of genetic breeding of a new cohort starts at a higher average level of $f_{i}$ compared to the previous period, the deterministic limit of the stochastic dynamics will also lead to a higher new average value two periods later compared to the period before. In the infinite population limit, this trend will continue until the entire population will have converged to $f_{i}(t)=1$. Although, in this situation, all inherent tendencies of genetic changes come to a halt, the first mutation of an individual leading to an $f_{i}<1$ will destabilize this stationary state again and generate a systematic downward trend which over time leads to a convergence of the whole population to $f_{i}(t)=0$. Here again, any mutation will exert a destabilizing tendency commencing the upward part of the cycle ... and so on ad infinitum. Note that this endlessly repeating cycle should also somehow exist as a tendency in the finite population case (since what we observe in the large population case should correspond to the mean value dynamics of small economies). Of course, the cyclical development shown in Fig. 4 would not be consistent with a unit root in log exchange rates (it is a clearly mean reverting process) and volatility clustering. In a sense, with a large population, the inherent randomness of the artificial economy gets lost and the measurable macroeconomic observables $\left(p_{i}(t), e(t)\right)$ become deterministic quantities.

However, at least for very small populations, this inherent structure of the combined genetic and economic process seems to be entirely concealed by the random elements in the genetic processes on the level of the individual. In fact, the systematic tendencies pointed out above will be subject to more random distortions with a small population size. An upward or downward tendency will be inverted as soon as the portfolio fraction of a new generation is not higher (lower) than that of the preceding one. It is the more likely that this random event happens the smaller the size of the population is. The apparently realistic time series characteristics result from situations where this happens with a very high probability within a few time steps. This explains why these more irregular dynamics with recurrent bursts of activity are only observed within a certain range of small numbers of agents.

We end our attempts at providing intuitive explanations of the evolutionary dynamics of our model with some remarks on the dynamics of second moments. As can be seen from Fig. 4, even the standard deviations of our choice variables exhibit predictable systematic patterns over the cycle. In particular, both the standard deviation of first-period consumption and the standard deviation of the fraction of domestic assets increase when one of the corner equilibria becomes unstable, remain relatively high during most of the motion to the opposite end of the parameter space and converge to zero when this new stationary solution is eventually approached. Note that this also implies that despite the near constancy of the mean value of $c_{i}(t)$, the dynamics is often characterized by a relatively wide range of individual choices. What happens is that after destabilization of an equilibrium, a broad range of choices of $c_{i}(t)$ and $f_{i}(t)$ gives higher utility (as can be inferred from the indifference curves in Fig. 5). Hence, many different types of mutations will be allowed to enter the population. The distribution of the choice variables spreads out and in the following, the whole population moves like a swarm from the left-hand side of the space of choice variables to its upper 


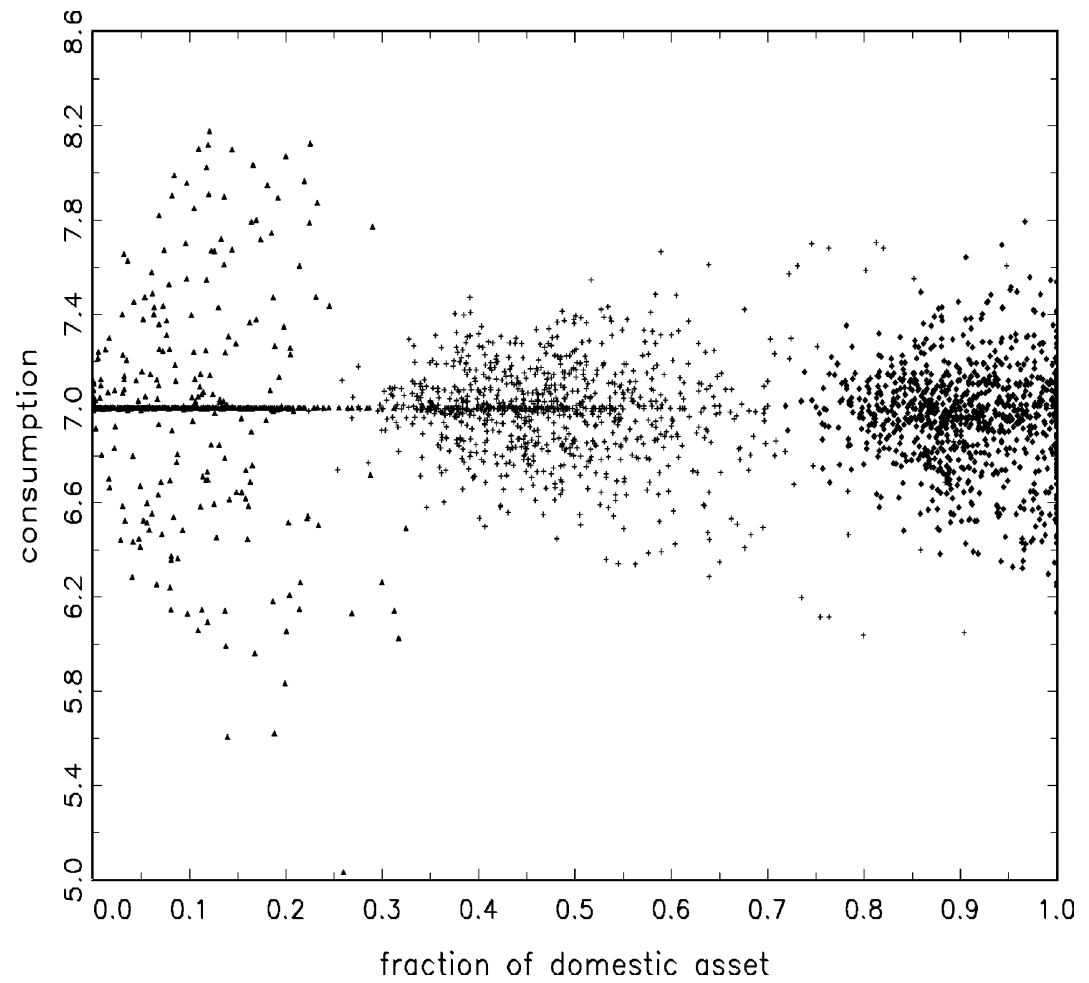

Fig. 6. A snapshot of the evolution of the population corresponding to one upward half-cycle in Fig. 4. The graph shows on its left-most part the distribution of choice parameters within a generation shortly after the lower turning point (triangles). The pluses and diamonds show the distribution of choice parameters within the same dynasty after 40 and 80 periods, respectively.

right-hand end (cf. Fig. 6). When the portfolio choices converge to a homogeneous situation $f_{i}(t)=1$ again, higher indifference curves can only be reached with consumption levels close to the steady state level $c^{*}$. This leads to a decrease of the bandwidth of first-period consumption levels in the population. Eventually, the variability with respect to both choice variables shrinks to zero. Once all individuals are close to the utility-maximizing steady state levels $\left(f_{i}(t)=1, c_{i}(t)=c^{*}\right)$, a small destabilizing mutation will lead to a sudden spread of the distribution of strategies and will set into motion a movement to the left of the whole swarm of individuals.

\section{Conclusions}

Elaborating on the GA version of the Kareken-Wallace model introduced by Arifovic (1996) and Arifovic and Gencay (2000), we have analyzed both the potential and the limitations for this type of artificial open economy to generate realistic time series properties. 
As it turns out, the model can generate time series which very closely mimic the statistical characteristics of empirical data. The mechanism responsible for the emergence of these interesting dynamics seems to be similar to the one analyzed within a different context by Lux and Marchesi (1999): the model has a continuum of equilibria with an indeterminate distribution of strategies among agents (as has been argued above, any distribution of the $f_{i}$ would be admissible in equilibrium). With the stochasticity of the genetic process, there will always be distortions preventing the system from settling at any particular equilibrium. Because of the evolutionary instability of any distribution of strategies these random distortions will evoke self-amplifying tendencies which produce large price changes (fat tails) and volatility clustering. However, we also find that a small probability of mutation and a small number of agents are needed to get this realistic output for the exchange rate. ${ }^{9}$ With a large population, the destabilizing tendencies are so strong that the crucial choice variable, $f_{i}$, bounces back and forth between the corners of the admissible parameter space. This applies to both binary and real-coded GAs. While the requirement of small mutation rates might be considered to be plausible and not too restrictive, having to restrict the population size to numbers below, say, $N=1000$ is much more cumbersome. Real markets (in particular, the world-wide market for foreign exchange), surely have more participants so that $N<1000$ seems an unrealistic requirement. However, this disappointing finding is shared by other multi-agent models (cf. Egenter et al., 1999; Yeh, 2001; Challet and Marsili, 2002). Essentially, with high N, a law of large numbers becomes effective even in models with a large number of available strategies and the randomness from the interaction between the microscopic choices of strategies vanishes. While in certain models, prices converge to fundamental values in the large economy limit (Egenter et al., 1999), the absence of fundamentals in the Kareken-Wallace model appears to be responsible for the oscillations between extreme choices.

How could one overcome these uncomfortable findings and save the 'nice' results obtained with smaller populations? One possibility would be to allow for more coherence among individuals via social sharing of information. Allowing for groups of agents to form, we would get a smaller effective number of agents. As an alternative, endogenous development of wealth could lead to some agents exerting more influence on the market outcome than others (of course, this feature would be particularly difficult to incorporate into the present simple model). This would presumable also change the outcome in a way that differs from the atomistic case analyzed above. ${ }^{10}$ Exploring these avenues is left for future research.

\footnotetext{
${ }^{9}$ Having shown the close similarity between empirical data and those from our artificial market at least in the case of a small population, it might be tempting to more formally estimate the parameters of the model. However, note that the very nature of financial returns data (non-existence of certain moments due to fat tails and long-term dependence of volatility) makes application of standard econometric tools (like estimation of parameters via Simulated Method of Moments) difficult.

${ }^{10}$ However, some preliminary simulations of an extended model showed that heterogeneity of wealth does not per se preserve the features of the small economy case: in a model with a heterogeneous distribution of endowments (following a Pareto law) the cyclic tendencies of the large economy case seemed to survive.
} 


\section{Acknowledgements}

The authors are grateful for many helpful comments by the participants of seminars at various universities as well as workshops and conferences at Trieste (WEHIA '02), Swiss Exchange Zurich (Workshop on Evolutionary Finance), Leiden (Workshop on Economic Dynamics), Aix-en-Provence (SCE Annual Conference '02), Los Angeles (Conference on 'Economic Behaviour and Organization') and the Bundesbank Training Centre at Eltville. Comments and suggestions by two referees and the editors-in-charge, Thorsten Hens and Klaus Schenk-Hoppé, are also gratefully acknowledged. T.L. also appreciates financial support by the Landeszentralbank Schleswig-Holstein and the Japan Society for the Promotion of Science. The penultimate version of this paper has been completed during his sabbatical stay at International Christian University Tokyo whose great hospitality is also gratefully acknowledged.

\section{References}

Arifovic, J., 1996. The behavior of the exchange rate in the genetic algorithm and experimental economies. Journal of Political Economy 104, 510-541.

Arifovic, J., Gencay, R., 2000. Statistical properties of genetic learning in a model of exchange rate. Journal of Economic Dynamics and Control 24, 981-1005.

Bilson, J., 1981. The speculative efficiency hypothesis. Journal of Business 54, 435-451.

Blume, L., Easley, D., 1992. Evolution and market behavior. Journal of Economic Theory 58, 9-40.

Bornholdt, S., 2001. Expectation bubbles in a spin model of markets: intermittency from frustration across scales. International Journal of Modern Physics C 12, 667-674.

Brock, W., Hommes, C., 1997. A rational route to randomness. Econometrica 65, 1059-1095.

Brock, W., Hommes, C., Wagener, F., 2003. Evolutionary dynamics in financial markets with many trader types, Journal of Mathematical Economics, this issue.

Challet, D., Marsili, M., 2002. Criticality and Finite-Size Effects in a Simple Realistic Model of Stock Market. Oxford University, Mimeo (http://xxx.lanl.gov/archive/cond-mat/0210549).

Chen, S.-H., Lux, T., Marchesi, M., 2001. Testing for nonlinear structure in an 'artificial' financial market, Journal of Economic Behavior and Organization 46, 327-342.

Chiarella, C., He, T., 2002. An Adaptive Model of Asset Pricing and Wealth Dynamics with Heterogeneous Trading Strategies. University of Technology Sydney, Mimeo.

Chiarella, C., He, T., 2001. Asset price and wealth dynamics under heterogeneous expectations. Quantitative Finance 1, 509-526.

Dacorogna, M., Gencay, R., Müller, U., Olsen R., Pictet, O., 2001. An Introduction to High-Frequency Finance. New York.

Dawid, H., 1999. Adaptive Learning by Genetic Algorithms: Analytical Results and Applications to Economic Models, second ed. Springer, Berlin.

De Vries, C., 1994. Stylized facts of nominal exchange rates. In: van der Ploeg, F. (Ed.), The Handbook of International Macroeconomics. Blackwell Publishers, Oxford.

Egenter, E., Lux, T., Stauffer, D., 1999. Finite-size effects in Monte Carlo simulations of two stock market models, Physica A 268, 250-256.

Eshelman, L., Schaffer, J., 1993. Real-coded genetic algorithms and interval schemata. In: Foundations of Genetic Algorithms 2. Morgan Kaufman Publishers, San Mateo.

Gaunersdorfer, A., Hommes, C., 2000. A Nonlinear Structural Model for Volatility Clustering. University of Vienna, Mimeo.

Geweke, J., Porter-Hudak, S., 1983. The estimation and application of long-memory time series models. Journal of Time Series Analysis 4, 221-238. 
Hens, T., Schenk-Hoppé, K., 2003. Evolution of portfolio rules in incomplete markets, Journal of Mathematical Economics, this issue.

Herrera, F., Lozano, M., Verdegay, J., 1998. Tackling real-coded genetic algorithms: operators and tools for behavioural analysis. Artificial Intelligence Review 12, 265-319.

Holland, J., 1975. Adaptation in Natural and Artificial Systems. University of Michigan Press, Ann Arbor.

Iori, G., 2002. A microsimulation of traders' activity in the stock market: the role of heterogeneity, agents' interactions and trade frictions. Journal of Economic Behavior and Organization 49, 269-285.

Kareken, J., Wallace, N., 1982. On the indeterminacy of equilibrium exchange rates. Quarterly Journal of Economics 96, 207-222.

Kirman, A., Teyssière, G., 2002. Microeconomic models for long memory in the volatility of financial time series. Studies in Nonlinear Dynamics and Econometrics 5, 137-156.

Lux, T., Marchesi, M., 1999. Scaling and criticality in a stochastic multi-agent model of a financial market. Nature 397, 498-500.

Lux, T., Marchesi, M., 2000. Volatility clustering in financial markets: a micro-simulation of interacting agents. International Journal of Theoretical and Applied Finance 3, 675-702.

Meese, R., Rogoff, K., 1983. Empirical exchange rate models of the seventies: do the fit out of sample. Journal of International Economics 14, 3-24.

Prügel-Bennett, A., Shapiro, J., 1994. Analysis of genetic algorithms using statistical mechanics. Physical Review Letters 72, 1305-1309.

Sandroni, A., 2003. Markets favor Bayesian models, Journal of Mathematical Economics, this issue.

Sargent, T., 1993. Bounded Rationality in Macroeconomics. University Press, Oxford.

Srinivas, M., Patnaik, L., 1996. Genetic search: analysis using fitness moments. IEEE Transactions on Knowledge and Data Engineering 8, 120-133.

Yeh, C.-H., 2001. The Influence of Market Size in an Artificial Stock Market: The Approach Based on Genetic Programming. I-Shou University, Mimeo. 